\title{
Strichartz Estimates on Asymptotically de Sitter Spaces
}

\author{
Dean Baskin
}

\begin{abstract}
In this article we prove a family of local (in time) weighted Strichartz estimates with derivative losses for the Klein-Gordon equation on asymptotically de Sitter spaces and provide a heuristic argument for the non-existence of a global dispersive estimate on these spaces. The weights in the estimates depend on the mass parameter and disappear in the "large mass" regime. We also provide an application of these estimates to establish small-data global existence for a class of semilinear equations on these spaces.
\end{abstract}

\section{Introduction}

In this paper we prove a family of local (in time) weighted Strichartz estimates with derivative losses for the Klein-Gordon equation on asymptotically de Sitter spaces, with the constants depending explicitly on the length of the time interval. We also provide a heuristic argument for the non-existence of a global dispersive estimate on these spaces. As an application we establish small-data global well-posedness for a class of defocusing semilinear wave equations in this context.

The main novelty of this paper is twofold. The first is that local Strichartz estimates with loss follow from the Fourier integral operator (i.e., Lagrangian distribution) representation of the propagator near the diagonal. In particular, though we do not show it here, our method extends to prove dispersive-type

\footnotetext{
The author is very grateful to Rafe Mazzeo and András Vasy for countless helpful discussions and for supervising the thesis on which much of this research is based. The author is also grateful to Jared Wunsch, Austin Ford, Jeremy Marzuola, and Terence Tao for helpful conversations, and to an anonymous referee for numerous suggestions to improve this paper. This research was partially supported by NSF grants DMS-0801226 and DMS-1103436.
} 
estimates near the diagonal for a somewhat larger class of Lagrangian distributions. The second is that our method works even in the presence of timedependent metrics (see also Tataru [22] or Smith [19] for wave-packet proofs of Strichartz estimates in a time-dependent setting).

Strichartz estimates are mixed $L^{p}$ (in time) and $L^{q}$ (in space) estimates that provide a measure of dispersion for the wave and Klein-Gordon equations. A version of these estimates was originally discovered by Strichartz [21] and first appeared in their modern form in works of Ginibre and Velo [7] (for the Schrödinger equation); Kapitanskiǔ [11] and Mockenhaupt et al. [17] (for the wave equation); and Pecher [18] (for the Klein-Gordon equation). These estimates have been instrumental in proving well-posedness for various semilinear dispersive equations. In the context of general relativity, Marzuola et al. [16] and Tohaneanu [24] established Strichartz estimates for the static Schwarzschild and the rotating Kerr black hole backgrounds, respectively. Yagdjian and Galstian [27] proved a family of Strichartz-type estimates on a model of de Sitter space.

We now describe Strichartz estimates for the wave and Klein-Gordon equations on Minkowski space $\mathbb{R} \times \mathbb{R}^{n}$. For allowable exponents $(p, q, s)$, a solution $u$ of $\square u=0$ or $\square u+u=0$ satisfies the following estimate:

$$
\begin{aligned}
& \left(\int_{0}^{T}\|u(t, \cdot)\|_{W^{1-s, q}}^{p}\right)^{1 / p}+\left(\int_{0}^{T}\left\|\partial_{t} u(t, \cdot)\right\|_{W^{-s, q}}^{p}\right)^{1 / p} \\
& \quad \lesssim\|u(0, \cdot)\|_{H^{1}}+\left\|\left(\partial_{t} u\right)(0, \cdot)\right\|_{L^{2}}
\end{aligned}
$$

A similar estimate holds for the inhomogeneous equation. If $T<\infty$, then the estimate is local, while if $T=\infty$ it is global. The allowable exponents $(p, q, s)$ must satisfy two conditions: the admissibility condition and the scaling condition. For the wave equation the conditions are as follows:

$$
\begin{gathered}
\frac{2}{p}+\frac{n-1}{q} \leq \frac{n-1}{2} \\
\frac{1}{p}+\frac{n}{q}=\frac{n}{2}-s
\end{gathered}
$$

(wave admissibility)

With the Klein-Gordon equation, the scaling condition is unchanged, while the admissibility condition is less restrictive:

$$
\frac{2}{p}+\frac{n}{q} \leq \frac{n}{2}
$$

(KG admissibility)

If $s$ is larger than the value indicated by the scaling condition, the Strichartz estimates are said to have a loss of derivatives. The difference seen in the Strichartz exponents between the wave and Klein-Gordon equation is largely a long-time effect. As seen below in Theorem 1.1, we obtain analogs of the wave exponents and not the Klein-Gordon exponents. This effect is due to the lack of a global dispersive estimate in our setting.

The asymptotically de Sitter spaces we consider in this paper are diffeomorphic to $\mathbb{R} \times Y$ for some compact $n$-dimensional manifold $Y$ and are 
equipped with metrics having the following form near $t=+\infty$ :

$$
-\mathrm{d} t^{2}+e^{2 t} h\left(e^{-t}, y, \mathrm{~d} y\right)
$$

Here $h$ is a smooth (as a function of $e^{-t}$ ) family of Riemannian metrics on $Y$. In other words, we consider metrics that are asymptotically de Sitter in a global sense, i.e., they have a spacelike infinity. Note that the de Sitter-Schwarzschild and de Sitter-Kerr black hole spacetimes do not fall into this class. A more precise description of the class of metrics considered is given in Sect. 2 .

The main result of this paper is the following Strichartz-type estimate for the operator $P(\lambda)=\square_{g}+\lambda$ (a dictionary of function spaces is found in Sect. 1.1):

Theorem 1.1. Suppose that $(X, g)$ is an asymptotically de Sitter space, $t_{0}$ is sufficiently large, $\lambda>0$ and $\alpha$ is such that $0 \leq \alpha<\sqrt{\lambda}$ if $\lambda \leq \frac{n^{2}}{4}$ and $\alpha=\frac{n}{2}$ if $\lambda>\frac{n^{2}}{4}$. Suppose further that $\epsilon>0$ and $u$ satisfies the homogeneous initial value problem:

$$
\begin{aligned}
P(\lambda) u & =0 \\
\left.\left(u, \partial_{t} u\right)\right|_{t=t_{0}} & =(\phi, \psi)
\end{aligned}
$$

The function $u$ then satisfies a uniform local Strichartz estimate:

$$
\begin{aligned}
& \|u\|_{e^{(n-2 \alpha)\left(t-t_{0}\right) / q} L^{p}\left(\left[t_{0}, t_{0}+T\right] ;{ }^{0} W^{1-s, q}\left(\mathrm{~d} k_{t}\right)\right)} \\
& \quad+\left\|\partial_{t} u\right\|_{e^{(n-2 \alpha)\left(t-t_{0}\right) / q} L^{p}\left(\left[t_{0}, t_{0}+T\right] ;{ }^{0} W^{-s, q}\left(\mathrm{~d} k_{t}\right)\right)} \\
& \leq C \max \left(T, e^{(n-2 \alpha) T / 2}\right)\|(\phi, \psi)\|_{H_{E}\left(t_{0}\right)}
\end{aligned}
$$

Here the constant is independent of $t_{0}$ and $T$, while $p, q \geq 2, q \neq \infty$, and $(p, q, s)$ satisfies the following:

$$
\begin{aligned}
\frac{2}{p}+\frac{n-1}{q} & \leq \frac{n-1}{2} \\
\frac{1}{p}+\frac{n}{q} & =\frac{n}{2}+\epsilon-s
\end{aligned}
$$

If, in addition, we know that $h$ is independent of $t$ for large $t$, then we may take $\epsilon=0$ above.

A similar theorem holds for the inhomogeneous problem:

Theorem 1.2. Suppose that $(X, g)$ is an asymptotically de Sitter space, $t_{0}$ is sufficiently large, $\lambda>0$ and $\alpha$ is such that $0 \leq \alpha<\sqrt{\lambda}$ if $\lambda \leq \frac{n^{2}}{4}$ and $\alpha=\frac{n}{2}$ if $\lambda>\frac{n^{2}}{4}$. Suppose further that $\epsilon>0$ and $u$ satisfies the inhomogeneous initial value problem:

$$
\begin{aligned}
P(\lambda) u & =f \\
\left.\left(u, \partial_{t} u\right)\right|_{t=t_{0}} & =(0,0)
\end{aligned}
$$


The function $u$ then satisfies a uniform local Strichartz estimate with constant independent of $t_{0}$ and $T$ :

$$
\begin{aligned}
\|u\|_{e^{(n-2 \alpha)\left(t-t_{0}\right) / q} L^{p}\left(\left[t_{0}, t_{0}+T\right] ;{ }^{0} W^{1-2 s, q}\left(\mathrm{~d} k_{t}\right)\right)} & +\left\|\partial_{t} u\right\|_{e^{(n-2 \alpha)\left(t-t_{0}\right) / q} L^{p}\left(\left[t_{0}, t_{0}+T\right] ;{ }^{\circ} W^{-2 s, q}\left(\mathrm{~d} k_{t}\right)\right)} \\
\leq & C e^{(n-2 \alpha) T / 2} \max \left(1, T^{\left(\frac{1}{q^{\prime}}-\frac{1}{q}\right)\left(\frac{n-1}{2}\right)}\right) \\
& \cdot\|f\|_{e^{(n-2 \alpha) t / q} L^{p^{\prime}}\left(\left[t_{0}, t_{0}+T\right] ; L^{q^{\prime}}\left(\mathrm{d} k_{t}\right)\right)}
\end{aligned}
$$

Here $p, q \geq 2, q \neq \infty$, and $(p, q, s)$ must satisfy the following:

$$
\begin{aligned}
\frac{2}{p}+\frac{n-1}{q} & \leq \frac{n-1}{2} \\
\frac{1}{p}+\frac{n}{q} & =\frac{n}{2}+\epsilon-s
\end{aligned}
$$

If $h$ is independent of $t$ for large $t$, we may take $\epsilon=0$ above.

The difference in behavior for $\lambda>\frac{n^{2}}{4}$ and $\lambda \leq \frac{n^{2}}{4}$ is due to the expanding nature of the spacetime, which prevents energy conservation (or even a constant global energy bound) for $0 \leq \lambda<\frac{n^{2}}{4}$. The non-sharpness of the weight is a defect of our energy estimates and is due to the need to estimate a pseudodifferential operator (which requires controlling an inhomogeneous $H^{1}$ norm rather than a homogeneous one). This leads to our inability to treat the $\lambda=0$ case.

The difference in behavior can also be seen in the work of Yagdjian and Galstian [27] on de Sitter space. In that work, the authors obtain an analog of the dispersive estimates we prove in Sect. 6, with an improved estimate in the "large mass" setting.

We believe that the loss of $\epsilon$ derivatives in Theorems 1.1 and 1.2 is an artifact of our method (which requires regularizing a Fourier integral operator). Indeed, the work of Yagdjian and Galstian [27] on de Sitter space contains no such losses. Further evidence for this belief is that we obtain the estimates without a loss when $h$ is independent of $t$ for large $t$. Removing the loss in the more general setting requires a nontrivial extension of the Littlewood-Paley theory and is left to a future paper.

We prove only local estimates because the fundamental solution does not decay along the light cone, as shown by the author [1]. This paper contains a sketch of why this non-decay should imply the non-existence of a global dispersive estimate. We are unsure whether the global Strichartz estimates can still hold.

The author has previously established [2] Strichartz estimates without loss and with better decay for the conformal value of the Klein-Gordon mass $\left(\lambda=\frac{n^{2}-1}{4}\right)$. With this parameter, global Strichartz estimates are conformally equivalent to local in time Strichartz estimates for the wave equation on a compact Lorentzian cylinder. In this current manuscript we do not recover those stronger estimates. 
The inhomogeneous estimate above is weaker than the homogeneous one, both in terms of the weight and in terms of the exponents. The main difference, however, is that the exponents for the inhomogeneous problem do not "decouple", i.e., the spatial exponents must be dual to each other. This is due to the non-static nature of asymptotically de Sitter spaces. Indeed, in the case of static spacetimes, the propagator forms a semigroup and thus the estimates for the inhomogeneous problem follow from those for the homogeneous problem (see, for example, the paper of Keel and Tao [12]). As the spacetimes considered here are non-static, we must use a separate argument to treat the solution operator for the inhomogeneous problem directly, leading to the requirement above that the exponents for the solution and the inhomogeneous term must be dual to each other.

As an application of the Strichartz estimates, we prove a small-data global existence result for a defocusing semilinear Klein-Gordon equation. For $\lambda>\frac{n^{2}}{4}$, we consider the following semilinear equation:

$$
\begin{aligned}
P(\lambda) u+f_{k}(u) & =0 \\
\left.\left(u, \partial_{t} u\right)\right|_{t=t_{0}} & =(\phi, \psi)
\end{aligned}
$$

Here $f_{k}$ is a smooth function and must satisfy five conditions:

$$
\begin{gathered}
\left|f_{k}(u)\right| \lesssim|u|^{k} \\
|u| \cdot\left|f_{k}^{\prime}(u)\right| \sim\left|f_{k}(u)\right| \\
f_{k}(u)-f_{k}^{\prime}(u) \cdot u \leq 0 \\
F_{k}(u)=\int_{0}^{u} f_{k}(v) \mathrm{d} v \geq 0 \\
F_{k}(u) \sim|u|^{k+1} \quad \text { for large }|u|
\end{gathered}
$$

Conditions (A3) and (A4) are imposed so that energy estimates work in our favor. Indeed, (A3) implies that $F_{k}(u)-\frac{1}{2} f_{k}(u) \cdot u \leq 0$. Moreover, Assumption (A5) allows us to control the $L^{k+1}$ norm of solutions in terms of the energy.

We prove the following theorem:

Theorem 1.3. Suppose $(X, g)$ is an asymptotically de Sitter space with $h$ independent of $t$ for large $t$. Suppose further that $\lambda>\frac{n^{2}}{4}$ and $k=1+\frac{4}{n-1}$. There is an $\epsilon>0$ so that there is a unique solution $u$ to Eq. (1.2) provided the initial data satisfy the following smallness condition (here $F_{k}(u)=\int_{0}^{v} f_{k}(v) \mathrm{d} v$ is a (positive) antiderivative of $f_{k}$ ):

$$
\|\phi\|_{H^{1}\left(Y_{t_{0}}\right)}+\|\psi\|_{L^{2}\left(Y_{t_{0}}\right)}+\int_{Y_{t_{0}}} F_{k}(\phi) \mathrm{d} k_{t_{0}}<\epsilon
$$

In this case the solution $u$ lies in the following $L^{p}$ space:

$$
u \in L_{\mathrm{loc}}^{k+1}\left(\left[t_{0}, \infty\right) ; L^{k+1}\left(Y_{t}\right)\right) .
$$

The assumptions on $\lambda$ and $f_{k}$ imply that solutions obey a global energy bound and that the energy is positive definite, while the assumption on $h$ 
allows us to use the estimates without loss. Even with these somewhat restrictive hypotheses, the standard energy method does not seem to work here, as the Sobolev inequality introduces an additional exponential term. Indeed, unless $p$ is large enough ( $p$ must be at least $\left.\frac{2 n}{n-2}\right)$, the inclusion $W^{1,2}\left(Y, \mathrm{~d} h_{t}\right) \rightarrow$ $L^{p}\left(Y, \mathrm{~d} h_{t}\right)$ yields an inclusion of the same form for $\left(Y, \mathrm{~d} k_{t}\right)$ but with an exponentially growing (in $t$ ) bound.

Yagdjian [26] studied a similar equation (with an exponentially decaying function multiplying the nonlinearity) on de Sitter space. In that work, the author also considers only the "large mass" setting $\left(\lambda \geq \frac{n^{2}}{4}\right)$ and obtains a global existence result for a family of nonlinearities. Our present work considers a larger family of spacetimes and removes the exponentially decaying coefficient, but at a cost of restricting our attention to a single power for the nonlinearity.

The proof of the Strichartz estimates relies on an energy estimate and a dispersive estimate. The dispersive estimate is obtained by analyzing the representation of the fundamental solution found by the author $[1,3]$. We appeal to the parametrix construction in those papers because the uniform local estimates do not follow immediately from a scaling argument, even in the setting where $h$ is independent of $t$ (except possibly in the $\lambda=0$ setting). The estimates for the homogeneous problem $(f=0)$ require only the behavior of the solution operator near the diagonal in $X \times X$. Obtaining long-time estimates for the inhomogeneous problem, however, requires the far-field behavior of the propagator and so needs most of the parametrix construction given in the author's previous paper. The proof of the inhomogeneous estimate is the only place in this paper where we use the full parametrix.

The main ingredients in the proof of the existence result for the semilinear equation are an energy estimate and a contraction mapping argument using the inhomogeneous Strichartz estimate. We do not prove existence for a wider range of powers because we do not have an inhomogeneous $L^{1} L^{2} \rightarrow L^{p} L^{q}$ Strichartz estimate. As mentioned earlier, this is due to the non-static nature of the spacetime, so that the exponents do not decouple in the inhomogeneous problem.

In Sects. 2 and 4 we review asymptotically de Sitter spaces and briefly describe the structure of the solution operator for the Klein-Gordon equation on these spaces. In Sect. 3, we establish the energy estimates, while in Sects. 5 and 6 we prove the local dispersive estimates and discuss the obstruction to a global dispersive estimate. Section 7 proves the Strichartz estimates, while the final section, Sect. 8, discusses the application to the semilinear problem.

\subsection{Notation}

Throughout this manuscript, $X$ denotes a compact $(n+1)$-dimensional manifold with boundary, and $x$ is a boundary defining function on $X$. The interior of $X$ is diffeomorphic to $\mathbb{R}_{t} \times Y$ for a compact $n$-dimensional manifold $Y$. We typically use $y$ to denote coordinates on $Y$ and use $(x, y)$ and $(t, y)$ to denote coordinate systems on $X$ or its interior. The functions $x$ and $t$ are related (for large $t$ ) by $x=e^{-t}$. 
We consider only large $t$ and small $x$. Correspondingly, when a constant is independent of $t_{0}$, we mean that it is independent of $t_{0}$ provided that $t_{0}$ is bounded away from $-\infty$.

The interior of $X$ is equipped with a Lorentzian metric $g$ having a prescribed form near future infinity:

$$
g=\frac{-\mathrm{d} x^{2}+h(x, y, \mathrm{~d} y)}{x^{2}}=-\mathrm{d} t^{2}+e^{2 t} h\left(e^{-t}, y, \mathrm{~d} y\right)
$$

Here $h$ is a smooth (in $x$ ) family of Riemannian metrics on $Y$. At times, we treat $h$ both as a function of $x$ and as a function of $t$.

We use $Y_{t}$ to denote individual level sets of the function $t$. Each $Y_{t}$ is diffeomorphic to $Y$ and inherits an induced Riemannian metric we call $k_{t}$. For large $t$, it is related to $h_{t}$ (the restriction of $h$ to $Y_{t}$ ) by $k_{t}=e^{2 t} h_{t}$. The volume forms of $h_{t}$ and $k_{t}$ are denoted $\mathrm{d} h_{t}$ and $\mathrm{d} k_{t}$, respectively, and are related by $\mathrm{d} k_{t}=e^{n t} \mathrm{~d} h_{t}$ for large $t$. We use $h_{x}$ and $k_{x}$ when we wish to emphasize that $h$ and $k$ are also smooth in $x$.

We denote by $\square_{g}$ the D'Alembertian of $g$. In coordinates $(x, y)$ and $(t, y)$ it is given by the following expressions:

$$
\begin{aligned}
\square_{g} & =\left(x \partial_{x}\right)^{2}-n x \partial_{x}+\frac{x \partial_{x} \sqrt{h_{x}}}{\sqrt{h_{x}}} x \partial_{x}+x^{2} \Delta_{h_{x}} \\
& =\partial_{t}^{2}+n \partial_{t}+\frac{\partial_{t} \sqrt{h_{t}}}{\sqrt{h_{t}}} \partial_{t}+e^{-2 t} \Delta_{h_{t}}
\end{aligned}
$$

Here $\Delta_{h_{x}}$ (respectively, $\Delta_{h_{t}}$ ) is the Laplacian of the metric $h_{x}$ (respectively, $h_{t}$ ) restricted to the level sets of $x$ or $t$. We adopt the convention that it has positive spectrum. We define $P(\lambda)=\square_{g}+\lambda$.

We typically consider the inhomogeneous linear initial value problem:

$$
\begin{aligned}
P(\lambda) u & =f \\
\left.\left(u, \partial_{t} u\right)\right|_{t=t_{0}} & =(\phi, \psi)
\end{aligned}
$$

The homogeneous "even" (or "odd") problem refers to the situation when $f=0$ and $\psi=0$ (or $\phi=0$ ). The inhomogeneous problem refers to the situation when both $\phi$ and $\psi$ vanish. We typically denote by $U_{v}\left(t, t_{0}\right)$ the solution operator for the "odd" homogeneous problem and by $U_{p}\left(t, t_{0}\right)$ the solution operator for the "even" problem.

We denote by $H_{E, \mu}(t)$ (defined in Sect. 3) the energy space on $Y_{t}$ with the following norm:

$$
\|(\phi, \psi)\|_{H_{E, \mu}(t)}^{2}=\frac{1}{2} \int_{Y_{t}}\left[\left|\nabla_{k_{t}} \phi\right|_{k_{t}}^{2}+|\psi|^{2}+\mu|\phi|^{2}\right] \mathrm{d} k_{t}
$$

We denote the space $H_{E, \lambda}(t)$ by $H_{E}(t)$.

We denote by ${ }^{0} W^{s, p}\left(\mathrm{~d} k_{t}\right)$ the $L^{p}$-based Sobolev space of order $s$, defined with respect to the metric $k_{t}$ (defined in Sect. 4.3). It is equipped with the following norm: 


$$
\|\phi\|_{{ }^{0} W^{s, p}\left(\mathrm{~d} k_{t}\right)}=\left(\int_{Y_{t}}\left|\left(1+\Delta_{k_{t}}\right)^{s / 2} \phi\right|^{p} \mathrm{~d} k_{t}\right)^{1 / p}
$$

For a (possibly constant) family of Banach spaces $Z(t)$, we use the term $L^{p}\left(\left[t_{0}, T\right] ; Z\right)$ to denote the space of $Z(t)$-valued functions $u$ on $\left[t_{0}, T\right]$ satisfying the following:

$$
\left(\int_{t_{0}}^{T}\|v(t)\|_{Z(t)}^{p} \mathrm{~d} t\right)^{1 / p}<\infty
$$

We provide a more precise characterization of this space in Sect. 7 .

The operators we consider are sums of distributions in three classes:

$$
I_{0}^{m}\left(X_{0}^{2} ; \Lambda_{1}\right), \quad \mathcal{A}_{\mathrm{phg}}^{\mathcal{F}} I^{m}\left(\widetilde{X}_{0}^{2} ; \mathrm{LC}\right), \quad \text { and } \quad \mathcal{A}_{\mathrm{phg}}^{\mathcal{F}}\left(\widetilde{X}_{0}^{2}\right)
$$

These spaces are recalled briefly in Sect. 4 .

\section{Asymptotically de Sitter Spaces}

In this section we describe de Sitter space and define the class of asymptotically de Sitter spaces we consider.

De Sitter space is the constant curvature spherically symmetric solution of the vacuum Einstein equations with a positive cosmological constant. It can be realized as the one-sheeted hyperboloid $\left\{-X_{0}^{2}+\sum_{i=1}^{n+1} X_{i}^{2}=1\right\}$ in $(n+2)$ dimensional Minkowski space and so is diffeomorphic to $\mathbb{R} \times \mathbb{S}^{n}$. It is equipped with coordinates $(\tau, \theta), \theta \in \mathbb{S}^{n}$, given implicitly as follows:

$$
\begin{aligned}
X_{0} & =\sinh \tau \\
X_{i} & =\theta_{i} \cosh \tau .
\end{aligned}
$$

In these coordinates, it inherits an induced metric:

$$
g_{\mathrm{dS}}=-\mathrm{d} \tau^{2}+(\cosh \tau)^{2} \mathrm{~d} \theta^{2}
$$

If we restrict our attention to large $\tau$, de Sitter space provides a model of a closed but expanding universe. If $T=e^{-\tau}$ near $\tau=+\infty$, then $\tau=+\infty$ is given by $T=0$ and the metric takes on the following form:

$$
g_{\mathrm{dS}}=\frac{-\mathrm{d} T^{2}+\frac{1}{4}\left(1+T^{2}\right) \mathrm{d} \theta^{2}}{T^{2}}
$$

In other words, $\left(\mathbb{R} \times \mathbb{S}^{n}, g_{\mathrm{dS}}\right)$ is conformally compact with a spacelike boundary at infinity.

The class of asymptotically de Sitter spaces considered in the current paper is the same class studied by Vasy [25]. In what follows and in the rest of the paper, $X$ is a compact $(n+1)$-dimensional manifold with boundary, and $x$ is a boundary defining function for $X$, i.e., $\partial X=\{x=0\}$ and $\left.d x\right|_{x=0} \neq 0$. 
Definition 2.1. $(X, g)$ is an asymptotically de Sitter space if $g$ is a Lorentzian metric on the interior $X^{\circ}$ of $X$, and, in a collar neighborhood $[0, \epsilon)_{x} \times \partial X$ of the boundary, $g$ has the following form:

$$
g=\frac{-\mathrm{d} x^{2}+h}{x^{2}}
$$

Here $h$ is a smoothly varying family of symmetric $(0,2)$ tensors on $X,\left.h\right|_{\partial X}$ is a section of $T^{*} \partial X \otimes T^{*} \partial X$ (rather than $\left.T_{\partial X}^{*} X \otimes T_{\partial X}^{*} X\right)$, and $\left.h\right|_{\partial X}$ is a Riemannian metric on $\partial X$.

Remark 2.2. Proposition 2.1 of Joshi and Sá Barreto [10] implies that this definition is equivalent to the requirement that, in a collar neighborhood $[0, \epsilon)_{x} \times \partial X$ of the boundary, $g$ has the following form:

$$
g=\frac{-\mathrm{d} x^{2}+h(x, y, \mathrm{~d} y)}{x^{2}}
$$

Here $h(x, y, \mathrm{~d} y)$ is a family of Riemannian metrics on $\partial X$.

We further impose two global assumptions:

(B1) The boundary can be written as a disjoint union $\partial X=Y_{+} \cup Y_{-}$, where each $Y_{ \pm}$is a union of connected components of $\partial X$.

(B2) Each nullbicharacteristic (or light ray) $\gamma(t)$ of $g$ tends to $Y_{ \pm}$as $t \rightarrow \pm \infty$, or vice versa.

Taken together, assumptions (B1) and (B2) imply the existence of a global function $T \in C^{\infty}(X)$ with $T= \pm 1$ on $Y_{ \pm}$and $d T$ everywhere timelike (see, for example the paper of Geroch [5]). (In other words, there is a smooth function with timelike derivative agreeing with $1-x$ near $Y_{+}$and $x-1$ near $Y_{-}$.) The existence of such a function implies that $X$ is diffeomorphic to $[-1,1] \times Y_{+}$ and that $\left(X^{\circ}, g\right)$ is a globally hyperbolic spacetime. For convenience we set $Y=Y_{+}$.

As $Y_{+}$represents future infinity, we typically work with a timelike foliation of $X^{\circ}$ given by a function $t$ taking values from $-\infty$ to $\infty$. We may take this foliation so that $t=\log x$ near $Y_{-}$and $t=-\log x$ near $Y_{+}$. We denote by $Y_{t}$ the leaves of this foliation, i.e., $Y_{t_{0}}=\left\{t=t_{0}\right\}$. We denote by $k_{t}$ the induced metric on $Y_{t}$, while $h_{t}$ denotes the restriction of the family of Riemannian metrics $h$ to $Y_{t}$. The volume forms of $k_{t}$ and $h_{t}$ are denoted $\mathrm{d} k_{t}$ and $\mathrm{d} h_{t}$, respectively. Near $Y_{+}$, the metrics are related by $k_{t}=e^{2 t} h_{t}$ and $\mathrm{d} k_{t}=e^{n t} \mathrm{~d} h_{t}$.

Near $Y_{+}$, we typically work with coordinates $(x, y)$ or coordinates $(t, y)$. The form of $\square_{g}$ in these coordinates is recorded in Eq. (1.3). We set $P(\lambda)=$ $\square_{g}+\lambda$. This convention is chosen so that $\lambda>\frac{n^{2}}{4}$ corresponds to positive mass.

\section{Energy Estimates}

In this section we prove a family of energy estimates for the Eq. (1.4).

For a fixed $\mu$, we define the norm for an energy space on the spacelike slice $Y_{t}$. 
Definition 3.1. For initial data $(\phi, \psi) \in C^{\infty}\left(Y_{t}\right) \times C^{\infty}\left(Y_{t}\right)$, we define its energy norm by the following expression:

$$
\|(\phi, \psi)\|_{H_{E, \mu}(t)}^{2}=\frac{1}{2} \int_{Y_{t}}\left[\left|\nabla_{k_{t}} \phi\right|_{k_{t}}^{2}+|\psi|^{2}+\mu|\phi|^{2}\right] \mathrm{d} k_{t}
$$

For $\mu \geq 0$, this is a positive definite norm on the orthogonal complement of the constant functions, while for $\mu>0$, this is a positive definite norm. We define the energy space $H_{E, \mu}(t)$ as the completion of $C^{\infty}\left(Y_{t}\right) \times C^{\infty}\left(Y_{t}\right)$ with respect to this norm. We denote by $H_{E}(t)$ the space $H_{E, \lambda}(t)$.

Remark 3.2. Note that for $\lambda=0$, the $H_{E}(t)$ norm of $(\phi, \psi)$ does not control the $L^{2}$ norm of $\phi$. For this reason we restrict our attention to the case when $\lambda>0$.

Remark 3.3. We later conjugate our operator by $e^{n t / 2}$ to obtain improved energy estimates when $\lambda>\frac{n^{2}}{4}$. This suggests that the natural energy spaces to consider bound $\partial_{t} u+\frac{n}{2} u$ rather than $\partial_{t} u$. However, in order to ensure that the energy spaces behave like standard Sobolev spaces under interpolation, we do not consider these more natural energy spaces at the present time.

We soon require the following lemma.

Lemma 3.4. For sufficiently large $t$, the commutator of $\Delta_{k_{t}}$ with $\partial_{t}$ has the following form:

$$
\left[\partial_{t}, \Delta_{k_{t}}\right]=-2 \Delta_{k_{t}}+e^{-3 t} Q
$$

Here $Q$ is a smoothly varying (in $x=e^{-t}$ ) family of second order differential operators. If $h$ is independent of $t$ for large $t$, then $Q=0$.

Proof. We first note that $\Delta_{h}$ is a family of second order differential operators on $Y$, varying smoothly in $x$ down to $x=0$. In particular, the commutator, $Q=-\left[\partial_{x}, \Delta_{h_{x}}\right]$, is a second order differential operator on $Y$ also varying smoothly in $x$ down to $x=0$. If $h$ is independent of $x$, then $Q=0$.

We now use that $\Delta_{k_{x}}=x^{2} \Delta_{h_{x}}$ and calculate:

$$
\left[x \partial_{x}, \Delta_{k_{x}}\right]=\left[x \partial_{x}, x^{2} \Delta_{h_{x}}\right]=2 \Delta_{k_{x}}-x^{3} Q
$$

Substituting $\partial_{t}=-x \partial_{x}$ and $x=e^{-t}$ finishes the calculation.

For $a \geq 0$ and $\mu>0$, we now prove an energy estimate for an operator $\tilde{P}(a, \mu)$ given by the following expression near $Y_{+}$:

$$
\tilde{P}(a, \mu)=\partial_{t}^{2}+\left(a+O\left(e^{-t}\right)\right) \partial_{t}+\Delta_{k_{t}}+\mu
$$

Here $O\left(e^{-t}\right)$ denotes a smooth function in $e^{-t}$ that is bounded by $C e^{-t}$ for some constant $C$.

Proposition 3.5. If $(X, g)$ is an asymptotically de Sitter space, $a \geq 0, \mu>0$, and $u$ is a smooth function on the interior of $X$, then $u$ satisfies the following energy estimate: 


$$
\begin{array}{r}
\left\|\left(u, \partial_{t} u\right)(t)\right\|_{H_{E, \mu}(t)} \leq C\left(e^{n\left(t-t_{0}\right) / 2}\left\|\left(u, \partial_{t} u\right)\left(t_{0}\right)\right\|_{H_{E, \mu}\left(t_{0}\right)}\right. \\
\left.+\int_{t_{0}}^{t}\|\tilde{P}(a, \mu) u\|_{L^{2}\left(\mathrm{~d} k_{s}\right)} e^{n(t-s) / 2} \mathrm{~d} s\right)
\end{array}
$$

Here the constant $C$ is independent of $t_{0}$.

Proof. We know from the local theory of hyperbolic equations that the bound holds away from infinity (see, for example, the book of Taylor [23]). We must thus only show the bound near infinity for initial data on a slice near infinity.

For convenience we assume that $u$ is real-valued and we use the relationship between the Laplacian and the gradient:

$$
\int_{Y_{t}}|\nabla u(t)|_{k_{t}}^{2} \mathrm{~d} k_{t}=\int_{Y_{t}} u(t) \Delta_{k_{t}} u(t) \mathrm{d} k_{t}
$$

We now compute using the form of the operator $\tilde{P}$.

$$
\begin{aligned}
\partial_{t} & \left\|\left(u, \partial_{t} u\right)(t)\right\|_{H_{E, \mu}(t)}^{2} \\
= & \int_{Y_{t}}\left(\partial_{t} u\right)\left(\partial_{t}^{2} u+\Delta_{k_{t}} u+\mu u\right) \mathrm{d} k_{t} \\
& +\frac{1}{2} \int_{Y_{t}}\left(\left|\nabla_{k_{t}} u\right|_{k_{t}}^{2}+\left|\partial_{t} u\right|^{2}+\mu|u|^{2}\right)\left(n+\frac{\partial_{t} \sqrt{h_{t}}}{\sqrt{h_{t}}}\right) \mathrm{d} k_{t} \\
& +\frac{1}{2} \int_{Y_{t}} u\left[\partial_{t}, \Delta_{k_{t}}\right] u \mathrm{~d} k_{t} \\
= & \left(n+O\left(e^{-t}\right)\right)\left\|\left(u, \partial_{t} u\right)(t)\right\|_{H_{E, \mu}(t)} \\
& +\frac{1}{2} \int_{Y_{t}} u\left[\partial_{t}, \Delta_{k_{t}}\right] u \mathrm{~d} k_{t}+\int_{Y_{t}}(\tilde{P}(a, \mu) u)\left(\partial_{t} u\right) \mathrm{d} k_{t} \\
& -\int_{Y_{t}}\left(\partial_{t} u\right)^{2}\left(a+O\left(e^{-t}\right)\right) \mathrm{d} k_{t}
\end{aligned}
$$

We now use the calculation in Lemma 3.4, the positivity of two of the above terms, and the fact that $\int_{Y_{t}} e^{-3 t} u Q u \mathrm{~d} k_{t}$ is controlled by the product of $C e^{-t}$ and $\left\|\left(u, \partial_{t} u\right)\right\|_{H_{E, \mu}(t)}$. After dividing by $2\left\|\left(u, \partial_{t} u\right)\right\|_{H_{E, \mu}(t)}$, we may conclude the following:

$$
\begin{aligned}
& \partial_{t}\left\|\left(u, \partial_{t} u\right)(t)\right\|_{H_{E, \mu}(t)} \\
& \quad \leq\left(n+O\left(e^{-t}\right)\right)\left\|\left(u, \partial_{t} u\right)(t)\right\|_{H_{E, \mu}(t)}+\|\tilde{P}(a, \mu) u\|_{L^{2}\left(Y, \mathrm{~d} k_{t}\right)}
\end{aligned}
$$

An application of Gronwall's inequality finishes the proof. 
We now apply the previous proposition to prove an energy estimate for solutions $u$ of Eq. (1.4).

Proposition 3.6. Suppose that $(X, g)$ is an asymptotically de Sitter space and $u$ is smooth on the interior of $X$. If $\lambda>0$ and $0 \leq \alpha<\sqrt{\lambda}$ for $\lambda \leq \frac{n^{2}}{4}$ or $\alpha=\frac{n}{2}$ for $\lambda>\frac{n^{2}}{4}$, then $u$ satisfies the following estimate:

$$
\begin{array}{r}
\left\|\left(u, \partial_{t} u\right)(t)\right\|_{H_{E}(t)} \leq C\left(e^{(n-2 \alpha)\left(t-t_{0}\right) / 2}\left\|\left(u, \partial_{t} u\right)\left(t_{0}\right)\right\|_{H_{E}\left(t_{0}\right)}\right. \\
\left.\quad+\int_{t_{0}}^{t} e^{(n-2 \alpha)(t-s) / 2}\|P(\lambda) u\|_{L^{2}\left(Y_{s}, \mathrm{~d} k_{s}\right)} \mathrm{d} s\right)
\end{array}
$$

Here the constant $C$ is independent of $t_{0}$ if $t_{0}$ is bounded away from $-\infty$.

Remark 3.7. Note that for $\lambda>\frac{n^{2}}{4}, e^{(n-2 \alpha) t / 2} \equiv 1$.

Proof. Set $v=e^{\alpha t} u, a=n-2 \alpha$, and $\mu=\lambda-\alpha^{2}$, so that $a \geq 0$ and $\mu>0$. Note that $v$ then satisfies $\tilde{P}(\alpha, \mu) v=e^{\alpha t} P(\lambda) u$. Proposition 3.5 implies the following estimate for $v$ :

$$
\begin{aligned}
& \left\|\left(v, \partial_{t} v\right)(t)\right\|_{H_{E, \mu}(t)} \leq C\left(e^{n\left(t-t_{0}\right) / 2}\left\|\left(v, \partial_{t} v\right)\left(t_{0}\right)\right\|_{H_{E, \mu}\left(t_{0}\right)}\right. \\
& \left.+\int_{t_{0}}^{t} e^{n(t-s) / 2}\|\tilde{P}(a, \mu) v\|_{L^{2}\left(Y_{s}, \mathrm{~d} k_{s}\right)} \mathrm{d} s\right)
\end{aligned}
$$

Because $\mu>0$, we have that $\left\|\left(v, \partial_{t} v\right)(t)\right\|_{H_{E, \mu}(t)}$ and $e^{\alpha t}\left\|\left(u, \partial_{t} u\right)(t)\right\|_{H_{E}(t)}$ are comparable. Dividing through by $e^{\alpha t}$ finishes the proof.

\section{The Solution Operator}

In this section we recall from the author's previous work [1] the description of the solution operator for the Cauchy problem (1.4). We use this description primarily to ensure uniformity in our estimates, as the non-static nature of the spacetime means that we cannot represent in general the solution operator as a function of a fixed Laplacian and so must use another representation of the solution to obtain uniform estimates.

The Schwartz kernel of the solution operator is a Lagrangian distribution on a compactification $\widetilde{X}_{0}^{2}$ of the interior of $X \times X$. We briefly describe the result below and refer the reader to the author's previous work for more details $[1,3]$. Because we are interested only in obtaining uniform local Strichartz estimates, the homogeneous problem requires only the behavior near the diagonal near infinity. We nevertheless include a fuller description of the Schwartz kernel for two reasons. First, it indicates why we limit our attention to uniform local 


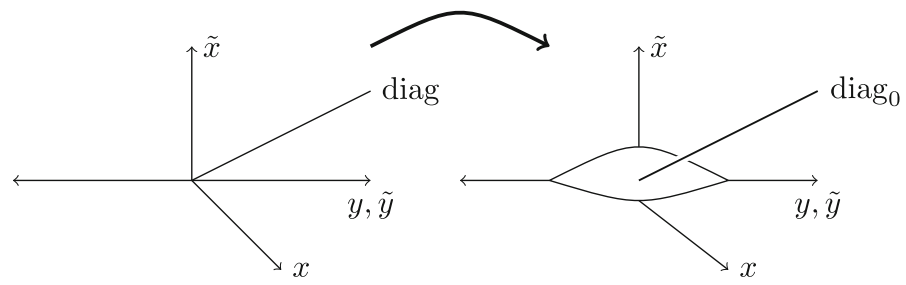

Figure 1. Passing from $X \times X$ to the 0 -double space $X_{0}^{2}=\left[X^{2}, \partial\right.$ (diag) $]$

Strichartz estimates - there is an obstruction ${ }^{1}$ to a global dispersive estimate that can be seen from the full parametrix. Second, the inhomogeneous problem typically requires an understanding of the behavior of the solution operator far from the diagonal. For static spacetimes, one may rely on the unitary group structure of the propagator to obtain estimates for the inhomogeneous problem in terms of those for the homogeneous one. In fact, this allows the Strichartz exponents for the inhomogeneous equation to "decouple" (so that the source and target exponents need not be dual to each other). The non-static nature of asymptotically de Sitter spaces, however, means that we must estimate the inhomogeneous solution operator directly.

\subsection{The Homogeneous Solution Operator}

For the homogeneous solution operators, we restrict our attention to the behavior of the Schwartz kernel near the diagonal and near infinity. In this region, the kernel is most easily described on $X_{0}^{2}$, a manifold with corners compactifying the interior of $X \times X$. This is the 0 -double space originally introduced by Mazzeo and Melrose [15].

We obtain $X_{0}^{2}$ by blowing up the boundary of the diagonal in $X \times X$, yielding a manifold with corners that has a new boundary hypersurface, which we call the front face ff. The lift of the diagonal and the flowout of the light cone to this manifold intersect all boundary hypersurfaces transversely. Figure 1 illustrates this blow-up.

The 0-double space has three boundary hypersurfaces: lf, the lift of the left face of $X \times X$ (given by $x=0$ in $X \times X$ ); rf, the lift of the right face (given by $\tilde{x}=0$ in $X \times X$ ); and ff, the front face introduced by the blow-up. Near the front face in a single coordinate chart for $Y$, the "polar coordinates" given below are smooth functions.

$$
r_{\mathrm{ff}}=\left(x^{2}+\tilde{x}^{2}+|y-\tilde{y}|^{2}\right)^{1 / 2}, \quad \theta=(x, y-\tilde{y}, \tilde{x}) / r_{\mathrm{ff}} \in \mathbb{S}^{n+1}
$$

It is often more convenient to work in projective coordinates near the front face away from $\mathrm{rf}$. These are given by the $(s, z, \tilde{x}, \tilde{y})$, where $s$ and $z$ are the following:

$$
s=\frac{x}{\tilde{x}}, \quad z=\frac{y-\tilde{y}}{\tilde{x}}
$$

The flowout by the Hamilton vector field of the principal symbol of $P$, $\sigma(P)$, of the characteristic set of $P$ intersected with the lifted diagonal is

\footnotetext{
1 The obstruction is described in Sect. 6.1.1.
} 
a smooth submanifold of $T^{*} X_{0}^{2}$ that intersects all boundary hypersurfaces transversely. This is a Lagrangian submanifold of $T^{*} X_{0}^{2}$, which we denote $\Lambda_{1}$. Near the front face but away from the diagonal, $\Lambda_{1}$ is the conormal bundle of a submanifold we call the light cone LC. Because we are only interested in the region near the front face, we may assume without loss of generality that LC is an embedded submanifold away from the diagonal.

In the following proposition, we summarize the relevant part of the construction in the author's previous work [1]. We denote by $I_{0}^{m}\left(X_{0}^{2} ; \Lambda_{1}\right)$ the space of Lagrangian distributions of order $m$ associated to the flowout Lagrangian $\Lambda_{1}$ and supported near the diagonal in $X_{0}^{2}$.

Proposition 4.1. Suppose that $t_{0}$ is large and that $U_{p}\left(t, t_{0}\right)$ and $U_{v}\left(t, t_{0}\right)$ are the solution operators for the "even" and "odd" homogeneous initial value problems, respectively. For any fixed $T_{0}$, let $K_{p}$ and $K_{v}$ be the distributional kernels of the operators $U_{p}$ and $U_{v}$, respectively, cut off to the region $\left|t-t_{0}\right| \leq T_{0}$. Here $K_{p}$ and $K_{v}$ should be regarded as distributions on $X \times X$. Under these conditions, $K_{p}$ and $K_{v}$ lie in the following spaces of Lagrangian distributions:

$$
\begin{aligned}
& K_{p} \in I_{0}^{-1 / 2}\left(X_{0}^{2} ; \Lambda_{1}\right) \\
& K_{v} \in I_{0}^{-3 / 2}\left(X_{0}^{2} ; \Lambda_{1}\right)
\end{aligned}
$$

\subsection{The Inhomogeneous Solution Operator}

Although we eventually use a representation of the solution of the inhomogeneous problem in terms of the solution operator of the homogeneous problem, we do use the extra information contained in this section. This is because the iterative process used to extend the homogeneous estimates in the Sect. 7.1 does not in general work for the inhomogeneous problem.

We consider the solution operator $E$ for the inhomogeneous problem:

$$
\begin{aligned}
P(\lambda) u & =f \\
\left.\left(u, \partial_{t} u\right)\right|_{t=t_{0}} & =(0,0)
\end{aligned}
$$

It can be written in terms of the homogeneous solution operator:

$$
(E f)(t)=\int_{t_{0}}^{t} U_{v}(t, s) f(s) \mathrm{d} s
$$

In the rest of this subsection we provide a fuller description of the Schwartz kernel of the operator $U_{v}(t, s)$ as $t, s \rightarrow+\infty$. More precisely, we describe the kernel of $U_{v}(t, s)$ considered as a distribution on $X \times X$.

The Schwartz kernel of $U_{v}(t, s)$ is a Lagrangian distribution on a compactification $\widetilde{X}_{0}^{2}$ of the interior of $X \times X$. It is obtained from $X_{0}^{2}$ by blowing up the intersection of the projection LC of $\Lambda_{1}$ with the side faces lf and $\mathrm{rf}$, introducing a new boundary hypersurface called the light cone face, or lcf. ${ }^{2}$ Figure 2 illustrates the space $\widetilde{X}_{0}^{2}$.

2 The full double space on which it lives is a bit more complicated, but we omit these complications here as we are only interested in the behavior near future infinity. We refer the reader to [1] for more details. 


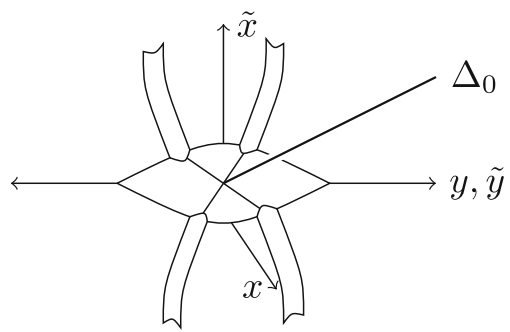

Figure 2. The double space $\widetilde{X}_{0}^{2}$ near ff

We now summarize the relevant result of the author's previous work [1]. As above, we denote by $I_{0}^{m}\left(X_{0}^{2} ; \Lambda_{1}\right)$ the space of Lagrangian distributions of order $m$ associated to the flowout Lagrangian $\Lambda_{1}$ and supported near the diagonal in $X_{0}^{2}$. We denote by $\mathcal{A}_{\text {phg }}^{\mathcal{F}} I^{m}\left(\widetilde{X}_{0}^{2} ; \mathrm{LC}\right)$ the space of distributions conormal to LC whose symbols have polyhomogeneous expansions with index family $\mathcal{F}$ at the side faces of $\widetilde{X}_{0}^{2}$. Finally, we denote by $\mathcal{A}_{\mathrm{phg}}^{\mathcal{F}}\left(\widetilde{X}_{0}^{2}\right)$ the space of polyhomogeneous conormal distributions with index family $\mathcal{F}$ on $\widetilde{X}_{0}^{2}$. For a precise definition of $\mathcal{A}_{\mathrm{phg}}^{\mathcal{F}} I^{m}\left(\widetilde{X}_{0}^{2} ; \mathrm{LC}\right)$, we refer the reader to [1]. For a discussion of polyhomogeneous conormal distributions, we refer the reader to a paper of Melrose [14].

Proposition 4.2. The kernel of the operator $U_{v}(t, s)$, regarded as a distribution on $X \times X$, can be written as a sum $K_{1}+K_{2}+K_{3}$, where $K_{i}$ are in the following spaces:

$$
\begin{aligned}
& K_{1} \in I_{0}^{-3 / 2}\left(X_{0}^{2} ; \Lambda_{1}\right) \\
& K_{2} \in \mathcal{A}_{\mathrm{phg}}^{\mathcal{F}} I^{-3 / 2}\left(\widetilde{X}_{0}^{2} ; \mathrm{LC}\right) \\
& K_{3} \in \mathcal{A}_{\mathrm{phg}}^{\mathcal{F}}\left(\widetilde{X}_{0}^{2}\right)
\end{aligned}
$$

Here $\Lambda_{1}$ is the flowout light cone, and the relevant index sets in the family $\mathcal{F}$ are given by the following:

$$
\begin{aligned}
& F_{\text {lcf }}=\left\{(j, \ell): \ell \leq j, j \in \mathbb{N}_{0}\right\} \\
& F_{\text {lf }}=F_{\text {rf }}=\left\{\left(\frac{n}{2} \pm \sqrt{\frac{n^{2}}{4}-\lambda}+m, 0\right): m \in \mathbb{N}_{0}\right\} \\
& F_{\text {ff }}=\left\{(m, 0): m \in \mathbb{N}_{0}\right\}
\end{aligned}
$$

\subsection{Regularization}

Our proof of the dispersive estimates in the general case rely on regularizing the solution operator. In this section we describe that regularization.

We rely on the notion of semiclassical pseudodifferential operators on the slices $Y_{t}$. Here the variable $\tilde{x}$ acts as the semiclassical parameter. In particular, we consider pseudodifferential operators with Schwartz kernels given by oscillatory integrals: 


$$
\frac{1}{(2 \pi \tilde{x})^{n}} \int_{\mathbb{R}^{n}} \mathrm{e}^{i(y-\tilde{y}) \cdot \eta / \tilde{x}} a\left(\frac{x}{\tilde{x}}, \frac{y-\tilde{y}}{\tilde{x}}, \tilde{x}, \tilde{y}, \eta\right) \mathrm{d} \eta
$$

Here $a$ is a symbol in $\eta$ and smooth in the rest of its arguments. We primarily use that powers of $1+\Delta_{k_{t}}$ are of this form.

We also use the boundedness of these operators on $L^{p}$ spaces. This is a standard result in semiclassical analysis.

Lemma 4.3. Suppose that $A_{x}$ is a family of pseudodifferential operators of order $-\epsilon$ on $Y$ of the form given by Eq. (4.1). If $1<p<\infty$, then $A_{x}$ is a bounded operator:

$$
A_{x}: L^{p}\left(Y, \mathrm{~d} k_{x}\right) \rightarrow L^{p}\left(Y ; \mathrm{d} k_{x}\right)
$$

Here the bound is independent of $x$.

Proof. We start by proving the same claim for $Y$ equipped with the volume form $\mathrm{d} h_{x}$.

Using a partition of unity, we write the symbol $a=a_{0}+a_{\infty}$, where $a_{0}$ is supported near the zero section and $a_{\infty}$ is supported away from 0 . The function $a_{0}$ is a Schwartz function in $\eta$, so we may use standard semiclassical results (see, e.g., Koch et al. [13]) to conclude that the bound holds for $a_{0}$.

For $a_{\infty}$, we appeal to Schur's test. Indeed, we must bound the integral over the left and right factors of $Y$, uniformly in $x$. Because $a_{\infty}$ is supported away from $\eta=0$, we may use the principle of non-stationary phase to bound the following integral:

$$
\int_{Y} \int_{\mathbb{R}^{n}} \mathrm{e}^{i\left(y-y^{\prime}\right) \cdot \eta / x} a_{\infty}\left(y, y^{\prime}, \eta\right) \mathrm{d} \eta \frac{\mathrm{d} y}{x^{n}}
$$

Indeed, integrating by parts $n$ times gives a bound of $O\left(x^{n}\right)$, which cancels the factor of $x^{-n}$ in the measure. A similar bound applies to the integral in the other factor, proving the claim.

This shows that $A_{x}$ is bounded $L^{p}\left(Y, \mathrm{~d} h_{x}\right) \rightarrow L^{p}\left(Y, \mathrm{~d} h_{x}\right)$. To prove that it is bounded $L^{p}\left(Y, \mathrm{~d} k_{x}\right) \rightarrow L^{p}\left(Y, \mathrm{~d} k_{x}\right)$, we note only that $A_{x}$ commutes with multiplication by $x$.

We require the following notion of $L^{p}$-based Sobolev spaces, which interpolate with the $L^{2}$-based Sobolev spaces in the standard way.

Definition 4.4. We define the ${ }^{0} W^{s, p}\left(\mathrm{~d} k_{t}\right)$ norm of a function $\phi \in C^{\infty}(Y)$ :

$$
\|\phi\|_{0 W^{s, p}\left(\mathrm{~d} k_{t}\right)}=\left(\int_{Y_{t}}\left|\left(1+\Delta_{k_{t}}\right)^{s / 2} \phi\right|^{p} \mathrm{~d} k_{t}\right)^{1 / p}
$$

We now prove a lemma that allows us to regularize the distributions in Sects. 4.1 and 4.2 .

Lemma 4.5. Suppose $K \in I_{0}^{m}\left(X_{0}^{2} ; \Lambda_{1}\right)$ is a Lagrangian distribution of order $m$ associated to $\Lambda_{1}$, and $A_{x} \in \Psi^{k}(Y)$ is a family of semiclassical pseudodifferential operators on $Y$ of order $k$, i.e., whose Schwartz kernels are given as 
oscillatory integrals of the form (4.1) with a a symbol of order $k$. The composition $A K$ is then also a Lagrangian distribution:

$$
A K \in I_{0}^{m+k}\left(X_{0}^{2} ; \Lambda_{1}\right)
$$

Here we may think of $A$ as acting on the left or the right $Y$ factor in $X \times X$. An analogous statement holds for $K \in \mathcal{A}_{\mathrm{phg}}^{\mathcal{F}} I^{m}\left(\widetilde{X}_{0}^{2} ; \Lambda_{1}\right)$.

Remark 4.6. Because $k_{x}=x^{-2} h$ near $x=0$, we may put $\left(1+\Delta_{k_{x}}\right)^{k / 2}$ in this form. The $x^{-n}$ in front of the oscillatory integral should be interpreted as coming from the half-density factors in the operator kernel.

Proof. Away from the front face, we may appeal to standard composition results. Although $A$ is not pseudodifferential on $X$, the additional wavefront set of the kernel of $A$ is disjoint from the operator wavefront set of $K$. Indeed, in local coordinates near $\partial(X \times X)$, it is contained in the following set:

$$
\left\{(x, y, x, \tilde{y}, \xi, 0,-\xi, 0):(y, \tilde{y}) \in \operatorname{supp} A_{x}\right\}
$$

The composition of this set with $\Lambda_{1}$ is empty, so we may microlocalize $A$ to be a pseudodifferential operator on $X$ without changing the singular structure of $A K$.

Near the front face, we use a different argument. Suppose that $A_{x}$ is a family of pseudodifferential operators on $Y$ with Schwartz kernels as in Eq. (4.1). Given a symbol $b(x, \tilde{x}, y, \tilde{y}, \xi)$ of order $m+\frac{1}{2}$, supported away from the side faces of $X_{0}^{2}$, we observe the following:

$$
A_{x}\left(b(x, \tilde{x}, y, \tilde{y}, \xi) \mathrm{e}^{i(y-\tilde{y}) \cdot \xi / x}\right)=c(x, \tilde{x}, y, \tilde{y}, \xi) \mathrm{e}^{i(y-\tilde{y}) \cdot \xi / x}
$$

Here $c$ is another symbol of order $k+m+\frac{1}{2}$. This can be seen (as in the book of Grigis and Sjöstrand [6]) via a careful application of stationary phase to the following integral:

$$
\int_{\mathbb{R}^{n}} \int_{Y_{x}} \mathrm{e}^{i\left(y-y^{\prime}\right) \cdot(\xi-\eta) / x} a\left(y, y^{\prime}, \eta\right) b\left(x, \tilde{x}, y^{\prime}, \tilde{y}, \xi\right) \frac{\mathrm{d} y^{\prime}}{x^{n}} \mathrm{~d} \eta
$$

The second statement follows by a similar argument.

\section{Dispersive Estimates for Lagrangian Distributions}

In this section we establish a family of uniform dispersive estimates for distributions in the same class as the wave propagator. In particular, we prove that 0-Lagrangian distributions associated to $\Lambda_{1}$ and supported near the diagonal obey a dispersive estimate. We note that the method we use to prove the dispersive estimate near the diagonal can be extended to a larger class of Lagrangian distributions.

Theorem 5.1. Suppose that $K \in I_{0}^{m}\left(X_{0}^{2} ; \Lambda_{1}\right)$ for $m=-\frac{n}{2}-1-\epsilon$ and $\epsilon>0$ and that $K$ is supported near the diagonal in a small neighborhood of the front 
face. Then in fact, in terms of coordinates $(s, z, \tilde{x}, \tilde{y}), K$ satisfies the following bound:

$$
|K| \lesssim|\log s|^{-(n-1) / 2+\epsilon}
$$

Moreover, because $|\log s| \lesssim 1$ on the support of $K$, we may ignore the $\epsilon$ in the previous bound.

Proof. We start by showing the dispersive estimate in the case where the Lagrangian $\Lambda_{1}$ is parametrized by the phase function $\phi_{0}$, given by the following in terms of $s$ and $z$ :

$$
\phi_{0}=z \cdot \zeta \pm(1-s)|\zeta|
$$

The argument is identical for either value of plus or minus, so we fix it to be plus. We assume that the distribution $K$ is supported near the front face. We may thus write it as an oscillatory integral of the following form:

$$
K=\int_{\mathbb{R}^{n}} \mathrm{e}^{i \phi_{0}} a(s, z, \tilde{x}, \tilde{y}, \zeta) \mathrm{d} \zeta
$$

Here $a$ is a symbol of order $m+\frac{1}{2}$. Using polar coordinates $\zeta=|\zeta| \hat{\zeta}$ and writing $\phi_{0}=(1-s)|\zeta|\left(\frac{z}{1-s} \cdot \hat{\zeta}+1\right)$, we may apply stationary phase to the above oscillatory integral:

$$
\begin{aligned}
|K| \lesssim & \int_{(1-s)^{-1}}|a(s, z, \tilde{x}, \tilde{y}, \pm|\zeta| \hat{z})| \\
& \cdot\left(|\zeta|^{(n-1) / 2}(1-s)^{-(n-1) / 2}+O\left(|\zeta|^{n-1 / 2}(1-s)^{-(n-2) / 2}\right)\right) \mathrm{d}|\zeta| \\
& +\int_{0}^{(1-s)^{-1}} \int_{\mathbb{S}^{n-1}}|a(s, z, \tilde{x}, \tilde{y},|\zeta| \hat{\zeta})||\zeta|^{n-1} \mathrm{~d} \hat{\zeta} \mathrm{d}|\zeta|
\end{aligned}
$$

The first term is bounded by $C(1-s)^{-(n-1) / 2+\epsilon}$ when $m=-\frac{n}{2}-1-\epsilon$. The second term within the parentheses is similarly bounded. We then bound the third term by the following expression:

$$
C+C \int_{1}^{(1-s)^{-1}}|\zeta|^{n+\frac{1}{2}+m} \mathrm{~d}|\zeta|
$$

This is then bounded by $C\left(1+(1-s)^{-(n-1) / 2+\epsilon}\right)$ when $m=-\frac{n}{2}-1-\epsilon$. Because this piece is supported near $s=1$, we may bound it by $(1-s)^{-(n-1) / 2+\epsilon}$.

We now use a perturbation argument to show that this estimate holds in a neighborhood of the front face for the Lagrangian $\Lambda_{1}$. Indeed, near the front face, $\Lambda_{1}$ may be parametrized by $\phi_{0}+|\zeta| r(s, z, \tilde{x}, \tilde{y}, \hat{\zeta})$, where $r=\tilde{x} b$ and $b$ is a smooth function of its arguments. For small enough $\tilde{x}, \phi$ is still a phase functions and its critical points (in $\hat{\zeta}$ ) are close to those of $\phi_{0}$. A similar argument to the one above thus shows that, in a small neighborhood of the front face, the same bound holds. 
Finally, because we are assuming that $K$ is supported near the diagonal, $(1-s) \sim \log s$ on the support of $K$. This finishes the proof.

We now prove a similar estimate for the larger class of distributions used for the inhomogeneous problem.

Lemma 5.2. Suppose that $K \in \mathcal{A}_{\mathrm{phg}}^{\mathcal{F}} I^{m}\left(\widetilde{X}_{0}^{2} ; \Lambda_{1}\right)$ with $m=-\frac{n}{2}-1-\epsilon$ and $\epsilon>0$. If $K$ is supported near the light cone $\mathrm{LC}$ and $F_{\mathrm{lcf}} \geq 0$, then $K$ satisfies the following bound when both $x$ and $\tilde{x}$ are close to 0 :

$$
|K| \lesssim \max \left(1,\left|\log \left(\frac{x}{\tilde{x}}\right)\right|^{-\frac{n-1}{2}+\epsilon}\right)
$$

Moreover, we may replace the exponent in the previous equation with $-\frac{n-1}{2}$.

Proof. Near the diagonal, the proof is identical to the proof of Lemma 5.1. Away from the diagonal, $\Lambda_{1}$ is the conormal bundle of an embedded submanifold and the phase function for this distribution is a perturbation of the following:

$$
\phi_{1}(s, z, \tilde{x}, \tilde{y}, \eta)=\frac{1}{s}((1-s)-|z|) \eta
$$

Here $s=x / \tilde{x}$ and $z=(y-\tilde{y}) / \tilde{x}$. The order $m$ is sufficiently negative that the symbol of the conormal distribution is integrable. We now simply combine the result near the diagonal with the symbol bound given from the order of polyhomogeneity.

We also record the following trivial but useful observation.

Lemma 5.3. If $K \in \mathcal{A}_{\mathrm{phg}}^{\mathcal{F}}\left(\widetilde{X}_{0}^{2}\right)$ with all index sets greater than or equal to 0 , then its Schwartz kernel is smooth on the interior of $X \times X$ and uniformly bounded on $X \times X$.

\section{Estimating the Propagator}

We now seek dispersive estimates for the propagator and for the solution operator for the inhomogeneous problem. We typically regularize the distribution, appeal to the result of the previous section to obtain a $L^{1} \rightarrow L^{\infty}$ type estimate, interpolate with an energy estimate, and then de-regularize.

\subsection{The Homogeneous Problem}

We start by fixing $t_{0}$ large. As before, we let $U_{v}\left(t, t_{0}\right)$ denote the solution operator for the "odd" problem and $U_{p}\left(t, t_{0}\right)$ denote the solution operator for the "even" problem. To simplify the calculation of adjoints, we consider the operators $\partial_{t} U_{v}\left(t, t_{0}\right),\left(1+\Delta_{k_{t}}\right)^{1 / 2} U_{v}\left(t, t_{0}\right), \partial_{t} U_{p}\left(t, t_{0}\right)\left(1+\Delta_{k_{t}}\right)^{-1 / 2}$, and $\left(1+\Delta_{k_{t}}\right)^{1 / 2} U_{p}\left(t, t_{0}\right)\left(1+\Delta_{k_{t}}\right)^{-1 / 2}$. All are elements of $I_{0}^{-1 / 2}\left(X_{0}^{2} ; \Lambda_{1}\right)$, and the energy estimates provide $L^{2} \rightarrow L^{2}$ bounds for them. We denote by $U_{\bullet}^{\prime}\left(t, t_{0}\right)$ any one of these operators. We seek a dispersive estimate for the products $U_{\bullet}^{\prime}\left(t, t_{0}\right) U_{\bullet}^{\prime}\left(s, t_{0}\right)^{*}$, where the adjoint is taken with respect to the $L^{2}$ inner product. 
We denote by $\tilde{U}_{\bullet}$ the regularization of $U_{\bullet}^{\prime}$ by order $r=\frac{n+1}{4}+\frac{\epsilon}{2}$, i.e., $\tilde{U}_{\bullet}=\left(1+\Delta_{k_{t}}\right)^{-r} U_{\bullet}^{\prime}$.

In order to estimate $\tilde{U}_{\bullet} \tilde{U}_{\bullet}^{*}$, we use the following lemma.

Lemma 6.1. Suppose that $s, t>t_{0}$ and $t_{0}$ is sufficiently large. For each $T_{0}$, the restriction of the product $\tilde{U}_{\bullet}\left(t, t_{0}\right) \tilde{U}_{\bullet}\left(s, t_{0}\right)^{*}$ to the neighborhood $\left|s-t_{0}\right| \leq T_{0}$ and $\left|t-t_{0}\right| \leq T_{0}$ is an element of $I_{0}^{-\frac{n}{2}-1-\epsilon}\left(X_{0}^{2} ; \Lambda_{1}\right)$, uniformly in $t_{0}$.

Proof. The Lagrangian submanifolds corresponding to $\tilde{U}_{\bullet}$ and $\tilde{U}_{\bullet}^{*}$ intersect transversely in $Y \times Y$, so we may follow the proof of Hörmander (Theorem 4.2.2 of [8]) to see that $\tilde{U}_{\bullet} \tilde{U}_{\bullet}^{*}$ is a Fourier integral operator. The uniformity follows from the smoothness of the distribution up to the front face.

The compositions have the stated order because restricting to $t^{\prime}=t_{0}$ shifts the order by $\frac{1}{4}$.

Combining the previous lemma with the results of Sect. 5 proves the following corollary.

Proposition 6.2. For $\epsilon>0, t_{0}$ large and $s, t$ within a fixed distance $T_{0}$ of $t_{0}$, the composition $\tilde{U}_{\bullet}\left(t, t_{0}\right) \tilde{U}_{\bullet}\left(s, t_{0}\right)^{*}$ is a bounded operator $L^{1}\left(\mathrm{~d} k_{s}\right) \rightarrow L^{\infty}\left(\mathrm{d} k_{t}\right)$ with the following bound:

$$
C|t-s|^{-\frac{n-1}{2}+\epsilon}
$$

Here the bound is independent of $t_{0}$.

Proof. An $L^{1}\left(\mathrm{~d} k_{s}\right) \rightarrow L^{\infty}\left(\mathrm{d} k_{t}\right)$ estimate is equivalent to a pointwise bound on $K$, where $K$ is the Schwartz kernel of the composition. The claim then follows in light of Lemmas 5.1 and 6.1. Indeed, the bound from Lemma 5.1 implies the following bound for $K$ (considered as an operator)::

$$
\|K \phi\|_{L^{\infty}\left(\mathrm{d} k_{x}\right)} \lesssim\left|\log \frac{x}{\tilde{x}}\right|^{-(n-1) / 2+\epsilon}\|\phi\|_{L^{1}\left(\mathrm{~d} k_{\tilde{x}}\right)}
$$

Changing coordinates from $(x, y)$ to $(t, y)$ finishes the proof.

By interpolating with the energy estimates in Sect. 3, we obtain the following family of dispersive estimates.

Theorem 6.3. Suppose that $\lambda>0, \epsilon>0$, and that $\alpha$ is such that $0 \leq \alpha<\sqrt{\lambda}$ if $\lambda \leq \frac{n^{2}}{4}$ and $\alpha=\frac{n}{2}$ if $\lambda>\frac{n^{2}}{4}$. If $t_{0}$ is sufficiently large and $\left|t-t_{0}\right|,\left|s-t_{0}\right| \leq T_{0}$ for a fixed $T_{0}$, then the composition $U_{\bullet}^{\prime}\left(t, t_{0}\right) U_{\bullet}^{\prime}\left(s, t_{0}\right)$ is a bounded operator between Sobolev spaces:

$$
{ }^{0} W^{\left(\frac{1}{q^{\prime}}-\frac{1}{q}\right)\left(\frac{n+1}{4}+\frac{\epsilon}{2}\right), q^{\prime}}\left(Y_{s}, \mathrm{~d} k_{s}\right) \rightarrow{ }^{0} W^{-\left(\frac{1}{q^{\prime}}-\frac{1}{q}\right)\left(\frac{n+1}{4}+\frac{\epsilon}{2}\right), q}\left(Y_{t}, \mathrm{~d} k_{t}\right)
$$

Here $q^{\prime}$ denotes the conjugate exponent of $q$ and ${ }^{0} W^{r, q}$ denotes the $L^{q}$-based Sobolev space of order $r$. As a map between these spaces, the operator obeys the following bound with $C$ independent of $t_{0}$ :

$$
C e^{(n-2 \alpha)\left(t-t_{0}\right) / q} e^{(n-2 \alpha)\left(s-t_{0}\right) / q}|t-s|^{-\left(\frac{1}{q^{\prime}}-\frac{1}{q}\right)\left(\frac{n-1}{2}\right)}
$$

Remark 6.4. If $\lambda>\frac{n^{2}}{4}$, the exponential terms in the bound above disappear. 
Proof. We may assume that $t, s>t_{0}$. Proposition 3.6 and the boundedness of pseudodifferential operators on $L^{2}$-based Sobolev spaces imply that $\tilde{U}_{\bullet}\left(t, t_{0}\right) \tilde{U}_{\bullet}\left(s, t_{0}\right)^{*}$ is a bounded operator:

$$
{ }^{0} W^{-\frac{n+1}{4}-\frac{\epsilon}{2}, 2}\left(Y_{s}, \mathrm{~d} k_{s}\right) \rightarrow{ }^{0} W^{\frac{n+1}{4}+\frac{\epsilon}{2}, 2}\left(Y_{t}, \mathrm{~d} k_{t}\right)
$$

As a map between these spaces, the operator is bounded by the following:

$$
C e^{(n-2 \alpha)\left(t-t_{0}\right) / 2} e^{(n-2 \alpha)\left(s-t_{0}\right) / 2}
$$

We now interpolate with the bounds in Proposition 6.2 to see that for $q \in(2, \infty)$, the composition $\tilde{U}_{\bullet}\left(t, t_{0}\right) \tilde{U}_{\bullet}\left(s, t_{0}\right)^{*}$ is a bounded operator:

$$
{ }^{0} W^{-\frac{2}{q}\left(\frac{n+1}{4}+\frac{\epsilon}{2}\right), q^{\prime}}\left(Y_{s}, \mathrm{~d} k_{s}\right) \rightarrow{ }^{0} W^{\frac{2}{q}\left(\frac{n+1}{4}+\frac{\epsilon}{2}\right), q}\left(Y_{t}, \mathrm{~d} k_{t}\right)
$$

As such a map, the operator has the following bound:

$$
C e^{(n-2 \alpha)\left(t-t_{0}\right) / q} e^{(n-2 \alpha)\left(s-t_{0}\right) / q}|t-s|^{-\left(\frac{1}{q^{\prime}}-\frac{1}{q}\right)\left(\frac{n-1}{2}-\epsilon\right)}
$$

We finally remove the regularization with Lemma 4.3. By ignoring the $\epsilon$ in the above bound, we may combine the losses from the estimate and the de-regularization into a single $\epsilon$.

If $h$ is independent of $t$ for large $t$, we may insert Littlewood-Paley projectors and remove the loss of regularity via now-standard arguments.

Theorem 6.5. Suppose that $\lambda>0, \epsilon>0$, and that $\alpha$ is such that $0 \leq \alpha<\sqrt{\lambda}$ if $\lambda \leq \frac{n^{2}}{4}$ and $\alpha=\frac{n}{2}$ if $\lambda>\frac{n^{2}}{4}$. If $t_{0}$ is sufficiently large and $\left|t-t_{0}\right|,\left|s-t_{0}\right| \leq T_{0}$ for a fixed $T_{0}$, then the composition $U_{\bullet}^{\prime}\left(t, t_{0}\right) U_{\bullet}^{\prime}\left(s, t_{0}\right)$ is a bounded operator between Sobolev spaces:

$$
{ }^{0} W^{\left(\frac{1}{q^{\prime}}-\frac{1}{q}\right)\left(\frac{n+1}{4}\right), q^{\prime}}\left(Y_{s}, \mathrm{~d} k_{s}\right) \rightarrow{ }^{0} W^{-\left(\frac{1}{q^{\prime}}-\frac{1}{q}\right)\left(\frac{n+1}{4}\right), q}\left(Y_{t}, \mathrm{~d} k_{t}\right)
$$

Here $q^{\prime}$ denotes the conjugate exponent of $q$. As a map between these spaces, the operator has the following bound with $C$ independent of $t_{0}$ :

$$
C e^{(n-2 \alpha)\left(t-t_{0}\right) / q} e^{(n-2 \alpha)\left(s-t_{0}\right) / q}|t-s|^{-\left(\frac{1}{q^{\prime}}-\frac{1}{q}\right)\left(\frac{n-1}{2}\right)}
$$

6.1.1. An Obstruction to a Global Dispersive Estimate. The non-decay of the principal symbol of the propagator near infinity provides an obstruction to a global dispersive estimate. In this section we briefly review how to obtain a global dispersive estimate on Minkowski space under our framework and then provide a heuristic argument for the lack of a global dispersive estimate on asymptotically de Sitter spaces. The argument we provide here is heuristic, but we believe it can be made rigorous by directly estimating solutions for the model problem on $\mathbb{R}_{s}^{+} \times \mathbb{R}_{z}^{n}$.

On Minkowski space $\mathbb{R} \times \mathbb{R}^{n}$, the propagator is given as a linear combination of terms of the following form:

$$
U(t, s, y, \tilde{y})=\int_{\mathbb{R}^{n}} \mathrm{e}^{i(y-\tilde{y}) \cdot \eta \pm i(t-s)|\eta|} a(y, \tilde{y}, t, s, \eta) \mathrm{d} \eta
$$

Here $a$ is a symbol of order 0 in $\eta$. The local dispersive estimate is known near the diagonal, so we assume here that $(t, s, y, \tilde{y})$ satisfy $|y-\tilde{y}|,|t-s| \geq 1$. Our 
goal is to write $U$ as a conormal distribution associated to the light cone and parametrized by a single variable. This is possible because the light cone is an embedded hypersurface away from the diagonal. To do this, we introduce modified polar coordinates in $\eta$ by writing $(t-s)|\eta|=\sigma$ and $\hat{\eta}=\frac{\eta}{|\eta|}$. Applying stationary phase in $\hat{\eta}$ shows that $U$ can be written in the following form:

$$
U(t, s, y, \tilde{y})=\int_{\mathbb{R}} \mathrm{e}^{i \rho(t, s, y, \tilde{y}) \sigma} \tilde{a}(t, s, y, \tilde{y}, \sigma) \mathrm{d} \sigma
$$

Here $\rho$ is a defining function for the light cone and $\tilde{a}$ is a symbol of order $\frac{n-1}{2}$ in $\sigma$ with symbol norms decaying as $|t-s|^{-\frac{n-1}{2}}$. In other words, $U$ is written in the desired form and its symbol exhibits the claimed decay.

Strictly speaking, however, the $W^{\frac{n-1}{2}, 1} \rightarrow L^{\infty}$ dispersive estimate does not hold even on Minkowski space, as the kernel of the propagator is unbounded. There are several ways to handle this minor defect. Three of the most popular include considering individually the spectral localizations of the solution, restricting the $L^{1}$-based Sobolev space to a Hardy space, and enlarging $L^{\infty}$ to a space of functions of bounded mean oscillation. Here we take a different approach. We consider instead the dispersive estimate satisfied by the regularization of the propagator by order $\frac{n+1}{2}+\epsilon$. In Minkowski space, the kernel of $\left(1+\Delta_{y}\right)^{-\frac{n+1}{2}-\epsilon} U(t, s, y, \tilde{y})$ has the same form as in Eq. (6.1) but with a symbol of order $-1-\epsilon$. The symbol of the regularized propagator is then integrable and exhibits decay of the requisite form, i.e., $\left(1+\Delta_{y}\right)^{-\frac{n+1}{2}-\epsilon} U(t, s)$ satisfies an $L^{1} \rightarrow L^{\infty}$ estimate on Minkowski space with bound $|t-s|^{-\frac{n-1}{2}}$.

A careful reading of Section 12 of the author's previous work [1] shows that the principal symbol of the propagator on an asymptotically de Sitter space (away from the diagonal) approaches a nonzero constant. Regularizing $U_{v}$ by order $\frac{n+1}{2}+\epsilon$ and applying the same argument shows that the regularized propagator has the following form near the intersection of the front face and the light cone face:

$$
\int_{\mathbb{R}} \mathrm{e}^{i \rho \eta} a\left(\rho, s, \frac{z}{|z|}, \tilde{x}, \tilde{y}, \eta\right) \mathrm{d} \eta
$$

Here $\rho=\frac{1-s-|z|}{s_{1+\epsilon}}$ and $a$ is an elliptic symbol (for each fixed $s$ ) of order $-1-\epsilon$ so that $\left(1+\eta^{2}\right)^{\frac{1+\epsilon}{2}} a$ approaches a nonzero constant (for large $\eta$ ) as $s \rightarrow 0$. This integral is the inverse Fourier transform of a symbol and so is a distribution conormal to $\rho=0$. Treating it as a family of distributions in $s$, the non-decay of the principal symbol implies that any pointwise bound the family satisfies should not decay in $s$. In particular, the regularized propagator satisfies an $L^{1} \rightarrow L^{\infty}$ bound but with a non-decaying constant.

Upgrading this heuristic argument to a rigorous one (i.e., saturating the above $L^{1} \rightarrow L^{\infty}$ bound) would require several steps. The first and simplest step would be to show that applying the regularized propagator to a series of $L^{1}$-normalized bump functions approximating the delta function indeed yields a solution of the form (6.2) for fixed $\tilde{x}$ and $\tilde{y}$. The difficult step is to then show 
that the integral (6.2) in fact does not decay in $s$, i.e., there are no miraculous cancellations. For generic elliptic symbols of order $-1-\epsilon$ this statement is true, but it seems difficult to rule out decay for all such $a$.

\subsection{The Inhomogeneous Problem}

In this section we prove the dispersive estimates for $U(t, s)$ that are needed to prove inhomogeneous Strichartz estimates on arbitrarily long intervals. Indeed, we use that the solution operator $E$ for the inhomogeneous problem is related to the homogeneous propagator via Duhamel's principle:

$$
(E f)(t)=\int_{t_{0}}^{t} U_{v}(t, s) f(s) \mathrm{d} s
$$

We prove the following theorem about the operators $\partial_{t} U_{v}$ and $(1+$ $\left.\Delta_{k_{t}}\right)^{1 / 2} U_{v}$.

Theorem 6.6. Suppose that $\lambda>0, \epsilon>0$, and $\alpha$ is such that $0 \leq \alpha<\sqrt{\lambda}$ for $\lambda \leq \frac{n^{2}}{4}$ and $\alpha=\frac{n}{2}$ for $\lambda>\frac{n^{2}}{4}$. If $t_{0}$ is sufficiently large and $t, s \geq t_{0}$, then $\partial_{t} U_{v}(t, s)$ and $\left(1+\Delta_{k_{t}}\right)^{1 / 2} U_{v}(t, s)$ are bounded operators between Sobolev spaces:

$$
L^{q^{\prime}}\left(\mathrm{d} k_{s}\right) \rightarrow{ }^{0} W^{-2 s, q}\left(\mathrm{~d} k_{t}\right)
$$

Here $q \in(2, \infty)$ and $q^{\prime}$ denotes the conjugate exponent of $q$. The regularity exponent $s$ is given by the following:

$$
2 s=\left(1-\frac{2}{q}\right)\left(\frac{n+1}{2}+\epsilon\right)
$$

As a map between these Sobolev spaces, $\partial_{t} U_{v}(t, s)$ and $\left(1+\Delta_{k_{t}}\right)^{1 / 2} U_{v}(t, s)$ obey the following bound with $C$ independent of $t_{0}$ :

$$
C e^{(n-2 \alpha)(t-s) / q} \max \left(|t-s|^{-\left(\frac{1}{q^{\prime}}-\frac{1}{q}\right)\left(\frac{n-1}{2}\right)}, 1\right)
$$

If $h$ is independent of $t$ for large $t$, we may take $\epsilon=0$.

Remark 6.7. The regularity exponent on the right side is less than or equal to 1 so long as $q$ satisfies the following:

$$
q \leq 2+\frac{4}{n-1+2 \epsilon}
$$

In particular, if $h$ is independent of $t$ for large $t, 2 s$ is no larger than 1 for $q \leq 2+\frac{4}{n-1}$.

Proof. We start by letting $\tilde{U}(t, s)$ be $\partial_{t} U_{v}(t, s)$ or $\left(1+\Delta_{k_{t}}\right)^{1 / 2} U_{v}(t, s)$ regularized by order $\frac{n+1}{2}+\epsilon$ :

$$
\begin{aligned}
& \tilde{U}(t, s)=\left(1+\Delta_{k_{t}}\right)^{-\frac{n+1}{2}-\epsilon} \partial_{t} U_{v}(t, s) \quad \text { or } \\
& \tilde{U}(t, s)=\left(1+\Delta_{k_{t}}\right)^{-\frac{n+1}{2}-\epsilon}\left(1+\Delta_{k_{t}}\right)^{1 / 2} U_{v}(t, s)
\end{aligned}
$$


Lemmas $5.1,5.2$, and 5.3 show that $\tilde{U}(t, s)$ is a bounded operator $L^{1}\left(\mathrm{~d} k_{s}\right) \rightarrow$ $L^{\infty}\left(\mathrm{d} k_{t}\right)$ with the following bound:

$$
C \max \left(|t-s|^{-\frac{n-1}{2}+\epsilon}, 1\right)
$$

(Note that we may choose to remove the $\epsilon$ in the above bound.)

Moreover, the energy estimates in Sect. 3 show that $\tilde{U}(t, s)$ is a bounded operator between $L^{2}$-based Sobolev spaces:

$$
L^{2}\left(\mathrm{~d} k_{s}\right) \rightarrow{ }^{0} W^{\frac{n+1}{2}+\epsilon, 2}\left(\mathrm{~d} k_{t}\right)
$$

For $0 \leq \alpha<\sqrt{\lambda}$ if $\lambda \leq n^{2} / 4$ and $\alpha=n / 2$ if $\lambda>n^{2} / 4$, the operator has a bound of the following form:

$$
C e^{(n-2 \alpha)(t-s) / 2}
$$

Interpolating the above two bounds and then de-regularizing yields that $\partial_{t} U_{v}(t, s)$ and $\left(1+\Delta_{k_{t}}\right)^{1 / 2} U_{v}(t, s)$ are bounded operators:

$$
L^{q^{\prime}}\left(\mathrm{d} k_{s}\right) \rightarrow{ }^{0} W^{-\left(\frac{1}{q^{\prime}}-\frac{1}{q}\right)\left(\frac{n+1}{2}+\epsilon\right), q}
$$

It obeys the following bound:

$$
C e^{(n-2 \alpha)(t-s) / q} \max \left(|t-s|^{-\left(\frac{1}{q^{\prime}}-\frac{1}{q}\right)\left(\frac{n-1}{2}+\epsilon\right)}, 1\right)
$$

Here $q \in(2, \infty), q^{\prime}$ is the dual exponent to $q$, and $\alpha$ is as above. (Note that $\frac{1}{q^{\prime}}-\frac{1}{q}=1-\frac{2}{q}$.)

When $h$ is independent of $t$ for large $t$, we may repeat the above argument, replacing the regularizers with fixed Littlewood-Paley projectors to avoid the losses.

\section{Strichartz Estimates}

In this section we prove uniform local Strichartz estimates. We treat the homogeneous and inhomogeneous problems for $\lambda>0$ separately.

Before proving the estimates, we define the $L^{p}(I ; Z(t))$ spaces.

Definition 7.1. Suppose that $I \subset \mathbb{R}$ is an interval and $Z(t)$ a family of Banach spaces on $I$. We define the $L^{p}(I ; Z(t))$ norm for a $Z$-valued function on $I$ by the following:

$$
\|v\|_{L^{p}(I ; Z)}=\left(\int_{I}\|v(t)\|_{Z(t)}^{p} \mathrm{~d} t\right)^{1 / p}
$$

If this norm is finite, we say that $v \in L^{p}(I ; Z)$. 


\subsection{The Homogeneous Problem}

In this section we prove Theorem 1.1.

Remark 7.2. Note that if $s \leq 1$, Theorem 1.1 yields an $L^{p} L^{q}$ estimate for $u$.

Proof. For convenience, we give the proof for the "odd" problem $(\phi=0)$. The proof for the full homogeneous problem is nearly identical. We first prove the estimates on an interval of length $T_{0}=1$ and then add them up to obtain the full bound.

We start by defining, for $\psi \in L^{2}\left(\mathrm{~d} k_{t_{0}}\right)$, the operator $T_{q}$ :

$$
T_{q} \psi=e^{-(n-2 \alpha)\left(t-t_{0}\right) / q} U^{\prime}\left(t, t_{0}\right) \psi
$$

In the above we set $U^{\prime}\left(t, t_{0}\right)=\partial_{t} U_{v}\left(t, t_{0}\right)$. Consider also the formal adjoint of $T_{q}$ considered as an operator $L^{2}\left(\mathrm{~d} k_{t_{0}}\right) \rightarrow L_{t}^{\infty} L^{2}\left(\mathrm{~d} k_{t}\right)$. For $F \in L_{t}^{1}\left(\left[t_{0}, t_{0}+\right.\right.$ $\left.\left.T_{0}\right] ; L^{2}\left(\mathrm{~d} k_{t}\right)\right)$, this operator is given by the following:

$$
T_{q}^{*} F=\int_{t_{0}}^{t_{0}+T_{0}} e^{-(n-2 \alpha)\left(t-t_{0}\right) / q} U^{\prime}\left(s, t_{0}\right)^{*} F(s) \mathrm{d} s
$$

In particular, the operator $T_{q} T_{q}^{*}$ is given by the following:

$$
\begin{aligned}
\left(T T^{*} F\right)(t) & =\int_{t_{0}}^{t_{0}+T_{0}} e^{-(n-2 \alpha)\left(t+s-2 t_{0}\right) / q} U^{\prime}\left(t, t_{0}\right) U^{\prime}\left(s, t_{0}\right)^{*} F(s) \mathrm{d} s \\
& =\int_{t_{0}}^{t_{0}+T_{0}} V(t, s) F(s) \mathrm{d} s
\end{aligned}
$$

Theorem 6.3 implies that $V(t, s)$ is bounded as an operator between the following spaces:

$$
{ }^{0} W^{\left(\frac{1}{q^{\prime}}-\frac{1}{q}\right)\left(\frac{n+1}{4}+\frac{\epsilon}{2}\right), q^{\prime}}\left(Y_{s}, \mathrm{~d} k_{s}\right) \rightarrow{ }^{0} W^{-\left(\frac{1}{q^{\prime}}-\frac{1}{q}\right)\left(\frac{n+1}{4}+\frac{\epsilon}{2}\right), q}\left(Y_{t}, \mathrm{~d} k_{t}\right)
$$

It has the bound $C|t-s|^{-\left(\frac{1}{q^{\prime}}-\frac{1}{q}\right)\left(\frac{n-1}{2}\right)}$.

We now apply the Hardy-Littlewood-Sobolev method of fractional integration (see, for example, the book of Stein [20]). In our setting, this states that convolution with $|t|^{-\alpha}$ is a bounded operator $L^{p}(\mathbb{R}) \rightarrow L^{r}(\mathbb{R})$, provided $\frac{1}{p}+\alpha=\frac{1}{r}+1$. In particular, $T T^{*}$ is a bounded operator:

$$
L^{p^{\prime}}\left(\left[t_{0}, t_{0}+T_{0}\right] ;{ }^{0} W^{s, q^{\prime}}\left(\mathrm{d} k_{s}\right)\right) \rightarrow L^{p}\left(\left[t_{0}, t_{0}+T_{0}\right] ;{ }^{0} W^{-s, q}\left(\mathrm{~d} k_{t}\right)\right)
$$

Here $p^{\prime}$ is the conjugate exponent to $p, q^{\prime}$ the dual exponent to $q$, and $p$ and $s$ are given by the following:

$$
\begin{aligned}
\frac{2}{p}+\frac{n-1}{q} & =\frac{n-1}{2} \\
\frac{2}{q}\left(\frac{n+1}{4}+\frac{\epsilon}{2}\right) & =\frac{n+1}{4}+\frac{\epsilon}{2}-s
\end{aligned}
$$


In other words, the theorem holds in the case where we have equality in Eq. (1.1) (with a slightly different $\epsilon$ ).

To prove the remaining estimates, we regularize $U^{\prime}\left(t, t_{0}\right)$ by order $\frac{n}{2}+\frac{\epsilon}{2}$ and then the resulting composition has an integrable symbol. This yields a bound of the following form (here $\tilde{U}^{\prime}$ is this regularization):

$$
\left\|\tilde{U}^{\prime}\left(t, t_{0}\right) \tilde{U}^{\prime}\left(s, t_{0}\right)^{*}\right\|_{L^{1}\left(\mathrm{~d} k_{s}\right) \rightarrow L^{\infty}\left(\mathrm{d} k_{t}\right)} \leq C
$$

Interpolating this bound with the energy estimate and then removing the regularization shows that, for all $2<q<\infty, U^{\prime}\left(t, t_{0}\right) U^{\prime}\left(s, t_{0}\right)^{*}$ is a bounded operator ${ }^{0} W^{s, q^{\prime}} \rightarrow{ }^{0} W^{-s, q}$ with bound $C e^{(n-2 \alpha)\left(t+s-2 t_{0}\right) / q}$ where $s$ satisfies the following:

$$
\frac{n}{q}+\frac{\epsilon}{q}=\frac{n}{2}+\frac{\epsilon}{2}-s
$$

In particular, $T_{q} T_{q}^{*}$ is then a bounded operator $L^{1} W^{s, q^{\prime}} \rightarrow L^{\infty} W^{-s, q}$. In other words, we have that the theorem holds for $p=\infty$. Interpolating these bounds with the previous ones proves half of the theorem in the "odd" case for intervals of length $T_{0}$.

To finish the proof in the "odd" case on intervals of length $T_{0}$, we may replace $U^{\prime}\left(t, t_{0}\right)=\partial_{t} U_{v}\left(t, t_{0}\right)$ with $\left(1+\Delta_{k_{t}}\right)^{1 / 2} U_{v}\left(t, t_{0}\right)$ without changing the proof. The estimates in the "even" case follow by replacing $U_{v}\left(t, t_{0}\right)$ with $U_{p}\left(t, t_{0}\right)\left(1+\Delta_{k_{t_{0}}}\right)^{-1 / 2}$.

The estimate on an interval of length $T$ follows by summing the estimates on intervals of length $T_{0}=1$ and using the energy estimates of Proposition 3.6.

The final statement of the theorem follows by repeating the above proof with the dispersive estimates of Theorem 6.5.

Remark 7.3. By inserting exponential (or even polynomial) weights, it is possible to extend the bounds to be global in time, but for weighted spaces. (One replaces $T_{q}$ with $T_{q}=e^{-(n+\alpha+\delta)\left(t-t_{0}\right) / q} U\left(t, t_{0}\right)$ in the above.)

\subsection{The Inhomogeneous Problem}

In this section we prove Theorem 1.2. Our approach to the inhomogeneous problem is similar, but complicated by our inability to "sum up" bounds on small intervals without incurring exponential penalties from the energy estimate when $\lambda \leq \frac{n^{2}}{4}$ or obtaining a term of the form $\|f\|_{L^{1} L^{2}}$ on the right side of the estimate.

Remark 7.4. We make several remarks about the statement of Theorem 1.2:

1. The estimates in Theorem 1.2 are weaker than what might be expected from the homogeneous case because we must account for the far-field behavior of the propagator.

2. We require the exponents $q$ and $q^{\prime}$ for the spatial parts of the estimate to be dual to each other because the propagator is no longer a unitary group and so we cannot write $U(t, s)=U\left(t, t_{0}\right) U\left(s, t_{0}\right)^{*}$. This prevents us both from deducing the inhomogeneous estimate directly from the homogeneous one and from "decoupling" the exponents in the inhomogeneous 
problem. The regularity exponent here looks slightly different from the one in the homogeneous setting ( $2 s$ versus $s$ ) for the same reason.

3. If $q<2+\frac{4}{n-1}$ we may guarantee that $1-2 s \leq 0$ by choosing $p$ and $\epsilon$ appropriately. This yields an $L^{p^{\prime}} L^{q^{\prime}} \rightarrow L^{p} L^{q}$ estimate.

We require a variant of the Christ-Kiselev lemma (first proved by Christ and Kiselev [4]), which we state now. The version we state is slightly different from those in the literature, but the proof given by Hassell et al. [9] remains valid when $X$ and $Y$ are replaced by smoothing varying families of Banach spaces $X(t)$ and $Y(t)$.

Lemma 7.5 (Christ-Kiselev Lemma [4], see [9] for this variant). Let $X(t)$ and $Y(t)$ be smoothly varying families of Banach spaces, and for all $s, t \in \mathbb{R}$, let $K(t, s): X(s) \rightarrow Y(t)$ be an operator-valued kernel from $X(s)$ to $Y(t)$. Suppose we have the following estimate for all $t_{0} \in \mathbb{R}$, some $1 \leq p<q \leq \infty$ and all $f \in L^{p}\left(\left(-\infty, t_{0}\right) ; X(t)\right):$

$$
\left\|\int_{s<t_{0}} K(t, s) f(s) \mathrm{d} s\right\|_{L^{q}\left(\left[t_{0}, \infty\right) ; Y(t)\right)} \leq A\|f\|_{L^{p}(\mathbb{R} ; X(t))}
$$

There is some constant $C$ (depending on $p$ and $q$ ) so that the following estimate holds:

$$
\left\|\int_{s<t} K(t, s) f(s) \mathrm{d} s\right\|_{L^{q}(\mathbb{R} ; Y(t))} \leq C A\|f\|_{L^{p}(\mathbb{R} ; X(t))}
$$

Moreover, the same type of estimate holds if $\mathbb{R}$ is replaced by a finite interval.

Proof of Theorem 1.2. The solution operator $E$ for the inhomogeneous problem is given by the following:

$$
(E f)(t)=\int_{t_{0}}^{t} U_{v}(t, s) f(s) \mathrm{d} s
$$

We seek estimates for $\partial_{t} E$ and $\left(1+\Delta_{k_{t}}\right)^{1 / 2} E$. For convenience we use $L$ to denote either $\partial_{t}$ or $\left(1+\Delta_{k_{t}}\right)^{1 / 2}$.

We fix $T>0$ and consider the operator $A$ given by the following integral:

$$
(A f)(t)=\int_{t_{0}}^{t_{0}+T} e^{-(n-2 \alpha) t / q} L U_{v}(t, s) e^{(n-2 \alpha) s / q} f(s) \mathrm{d} s
$$

The dispersive estimate in Theorem 6.6 and the Hardy-Littlewood-Sobolev method of fractional integration show that $A$ is a bounded operator between the following spaces:

$$
L^{p^{\prime}}\left(\left[t_{0}, t_{0}+T\right] ; L^{q^{\prime}}\left(\mathrm{d} k_{t}\right)\right) \rightarrow L^{r}\left(\left[t_{0}, t_{0}+T\right] ;{ }^{0} W^{-2 s, q}\left(\mathrm{~d} k_{t}\right)\right)
$$


Here $\frac{1}{r}+1=\frac{1}{p^{\prime}}+\frac{n-1}{2}\left(\frac{1}{q^{\prime}}-\frac{1}{q}\right), 2 s=\left(1-\frac{2}{q}\right)\left(\frac{n+1}{2}+\epsilon\right)$, and the bound is $C T^{\frac{n-1}{2}\left(1-\frac{2}{q}\right)}$ (and independent of $t_{0}$ ).

The Christ-Kiselev lemma (Lemma 7.5) then shows that the operator $E_{0}$ is bounded as an operator between the same spaces, where $E_{0}$ is given by the following:

$$
\left(E_{0} f\right)(t)=\int_{t_{0}}^{t} e^{-(n-2 \alpha) t / q} L U_{v}(t, s) e^{(n-2 \alpha) s / q} f(s) \mathrm{d} s
$$

The operator $L E$ is related to $E_{0}$ in the following way:

$$
(L E f)(t)=e^{(n-2 \alpha) t / q} L E_{0}\left(e^{-(n-2 \alpha) s / q} f(s)\right)(t)
$$

The operator $L E$ is thus bounded between the following weighted spaces with the same bound and with all exponents as above:

$$
\begin{aligned}
& e^{(n-2 \alpha) t / q} L^{p^{\prime}}\left(\left[t_{0}, t_{0}+T\right] ; L^{q^{\prime}}\left(\mathrm{d} k_{t}\right)\right) \\
& \quad \rightarrow e^{(n-2 \alpha) t / q} L^{r}\left(\left[t_{0}, t_{0}+T\right] ;{ }^{0} W^{-2 s, q}\left(\mathrm{~d} k_{t}\right)\right)
\end{aligned}
$$

If we demand that $r=p$, the dual exponent of $p^{\prime}$, we must have that $p$ and $q$ are related:

$$
\frac{2}{p}+\frac{n-1}{q}=\frac{n-1}{2}
$$

A similar argument to the one in the proof of Theorem 1.1 (regularizing by order $n+\epsilon$ and then interpolating) finishes the proof of the main estimate. The statement for $h$ independent of $t$ follows by using the improved estimates in Theorem 6.6.

Remark 7.6. Note that in the above, if $\lambda>\frac{n^{2}}{4}$, we could in fact "sum up" the bounds on small intervals by using the better energy estimate. This would allow us to strengthen the bound in this case to $C \max (1, T)$, but at a cost of including the $L^{1} L^{2}$ norm of $f$.

\section{An Application to a Semilinear Equation}

In this section we prove Theorem 1.3, the application of the Strichartz estimates to a class of semilinear Klein-Gordon equations with $\lambda>\frac{n^{2}}{4}$. In particular, we assume $h$ is independent of $t$ for large $t$ and consider Eq. (1.2) with $k=1+\frac{4}{n-1}$.

We start by proving an energy estimate for solutions of the semilinear equation (1.2). Recall that $F_{k}(u)=\int_{0}^{u} f_{k}(v) \mathrm{d} v$ is a (positive) antiderivative of $f_{k}$.

Proposition 8.1. Suppose that $\lambda>\frac{n^{2}}{4}$ and that $u$ is a solution of the semilinear equation (1.2). There is a constant $C$ (independent of $t_{0}$ ) so that the following 
energy estimate holds:

$$
\begin{aligned}
E(t) & =\frac{1}{2} \int_{Y_{t}}\left[\left|\partial_{t} u(t)\right|^{2}+\left|\nabla_{k_{t}} u(t)\right|_{k_{t}}^{2}+\lambda|u(t)|^{2}\right] \mathrm{d} k_{t}+\int_{Y_{t}} F_{k}(u(t)) \mathrm{d} k_{t} \\
& \leq C E\left(t_{0}\right)
\end{aligned}
$$

Proof. Consider the function $v=e^{n t / 2} u$. The function $v$ satisfies the following semilinear equation (in the notation of Sect. 3):

$$
\tilde{P}\left(0, \lambda-\frac{n^{2}}{4}\right) v=-e^{n t / 2} f_{k}\left(e^{-n t / 2} v\right)
$$

Consider now the energy $\tilde{E}(t)$ of the function $v$ :

$$
\begin{aligned}
\tilde{E}(t)= & \frac{1}{2} \int_{Y_{t}}\left[\left|\partial_{t} v\right|^{2}+\left|\nabla_{k_{t}} v\right|_{k_{t}}^{2}+\left(\lambda-\frac{n^{2}}{4}\right)|v|^{2}\right] \mathrm{d} k_{t} \\
& +\int_{Y_{t}} e^{n t} F_{k}\left(e^{-n t / 2} v\right) \mathrm{d} k_{t}
\end{aligned}
$$

We now observe that Assumption (A3) above on our nonlinearity implies the following:

$$
\begin{aligned}
\partial_{t} \int_{Y_{t}} e^{n t / 2} F_{k}\left(e^{-n t / 2} v\right) \mathrm{d} k_{t} \\
=\int_{Y_{t}}\left(e^{n t / 2} f_{k}\left(e^{-n t / 2} v\right) \partial_{t} v\right) \mathrm{d} k_{t} \\
\quad+\int_{Y_{t}}\left(n e^{n t} F_{k}\left(e^{-n t / 2} v\right)-\frac{n}{2} e^{n t} f_{k}\left(e^{-n t / 2} v\right) e^{-n t / 2} v\right) \mathrm{d} k_{t} \\
\leq \int_{Y_{t}} e^{n t / 2} f_{k}\left(e^{-n t / 2} v\right) \partial_{t} v \mathrm{~d} k_{t}
\end{aligned}
$$

The methods of Sect. 3 then imply that there is a constant $C$ so that $\tilde{E}(t) \leq$ $C e^{n t} \tilde{E}\left(t_{0}\right)$.

We now use that $u=e^{-n t / 2} v$, together with $\lambda>\frac{n^{2}}{4}$ to conclude that there is a constant $C^{\prime}$ (independent of $t$ ) so that the following estimate holds:

$$
\frac{1}{C^{\prime}} e^{n t} E(t) \leq \tilde{E}(t) \leq C^{\prime} e^{n t} E(t)
$$

Using the energy bound for $v$ and dividing by $e^{n t}$ finishes the proof.

Proof of Theorem 1.3. We start by noting that Theorem 1.1, together with $k=1+\frac{4}{n-1}$, implies a bound for the solution $u$ of the homogeneous problem:

$$
\begin{aligned}
P(\lambda) u & =0 \\
\left.\left(u, \partial_{t} u\right)\right|_{t=t_{0}} & =(\phi, \psi)
\end{aligned}
$$


The bound is the following:

$$
\|u\|_{L^{k+1}\left(\left[t_{0}, t_{0}+T\right] ; L^{k+1}\left(\mathrm{~d} k_{t}\right)\right)} \leq C(1+T)\|(\phi, \psi)\|_{H_{E}\left(t_{0}\right)}
$$

Here $C$ is independent of $t_{0}$ and $T$. Similarly, Theorem 1.2 implies a bound for the solution $u$ of the inhomogeneous equation:

$$
\begin{aligned}
P(\lambda) u & =f, \\
\left.\left(u, \partial_{t} u\right)\right|_{t=t_{0}} & =(0,0)
\end{aligned}
$$

Indeed, $u$ satisfies the following:

$$
\|u\|_{L^{k+1}\left(\left[t_{0}, t_{0}+T\right] ; L^{k+1}\left(\mathrm{~d} k_{t}\right)\right)} \leq C\left(1+T^{r}\right)^{1 / 2}\|f\|_{L^{\frac{k+1}{k}}\left(\left[t_{0}, t_{0}+T\right] ; L \frac{k+1}{k}\left(\mathrm{~d} k_{t}\right)\right)}
$$

Here $r$ is positive and again the constant is independent of $t_{0}$ and $T$. Note that we have used here that $h$ is independent of $t$ for large $t$ so that the lossless bounds are available.

Suppose now that $\phi$ and $\psi$ satisfy the following bound:

$$
\|(\phi, \psi)\|_{H_{E}\left(t_{0}\right)}+\|\phi\|_{L^{k+1}\left(\mathrm{~d} k_{t_{0}}\right)} \leq M
$$

Note that we may control the $L^{k+1}$ norm of $\phi$ by considering where $\phi$ is large $(\geq 1)$ and small $(\leq 1)$. Assumption (A5) implies that the norm where $\phi$ is large is bounded by the nonlinear part of the energy while the part where $\phi$ is small is bounded by the $L^{2}$ norm of $\phi$ (which is in turn controlled by the energy because $\lambda>\frac{n^{2}}{4}$ ). This observation and the energy estimate in Proposition 8.1 then imply that if $u$ is a solution of Eq. (1.2) with initial data $(\phi, \psi)$, then there is a constant $A$ so that for all $t \geq t_{0}$, the following bound holds:

$$
\left\|\left(u(t), \partial_{t} u(t)\right)\right\|_{H_{E}(t)}+\|u(t)\|_{L^{k+1}\left(\mathrm{~d} k_{t}\right)} \leq A M
$$

The rest of the proof is now standard but is included here for completeness. We establish a solution on a time interval of length $T$ via the contraction mapping principle, and then use the energy estimates and the independence of the constants on $t_{0}$ to extend the solution.

We start by finding a solution on an interval $\left[t_{0}, t_{0}+T\right]$. We seek a fixed point of the following map:

$$
\mathcal{F} u(t)=\mathcal{S}(t)(\phi, \psi)+\mathcal{G}\left(f_{k}(u)\right)(t)
$$

Here $\mathcal{S}$ is the solution operator for the homogeneous problem and $\mathcal{G}$ is the solution operator for the inhomogeneous problem with zero initial data. The main estimate used is the following:

$$
\begin{gathered}
\left\|f_{k}(u)-f_{k}(v)\right\|_{L^{\frac{k+1}{k}}}\left(\left[t_{0}, t_{0}+T\right] ; L^{\frac{k+1}{k}}\left(\mathrm{~d} k_{t}\right)\right) \\
\leq C\|u-v\|_{L^{k+1} L^{k+1}}\||u|+|v|\|_{L^{k+1} L^{k+1}}^{k-1}
\end{gathered}
$$

This estimate follows from the mean value theorem and Assumption (A2). Suppose now that $\phi$ and $\psi$ satisfy the following smallness condition:

$$
\|(\phi, \psi)\|_{H_{E}\left(t_{0}\right)}+\|\phi\|_{L^{k+1}\left(\mathrm{~d} k_{t_{0}}\right)} \leq A \epsilon
$$


The estimates above show that if $\|u\|_{L^{k+1} L^{k+1}} \leq K \epsilon$, then $v=\mathcal{F} u$ satisfies the following:

$$
\|v\|_{L^{k+1} L^{k+1}} \leq C\left(1+T^{r}\right) A \epsilon+C^{\prime}\left(1+T^{r}\right)^{1 / 2}(K \epsilon)^{k}
$$

In particular, if $\epsilon$ is small enough, we may arrange that $\|v\|_{L^{k+1} L^{k+1}} \leq K \epsilon$ as well.

The contraction mapping procedure continues as follows. Let $u^{(-1)}=0$ and $u^{(0)}$ be the solution of the homogeneous problem with initial data $(\phi, \psi)$. For $m>0$, let $u^{(m)}=\mathcal{F} u^{(m-1)}$. The above estimates then imply that there is new constant $C$ so that the following estimate holds:

$$
\begin{aligned}
& \left\|u^{(m+1)}-u^{(m)}\right\|_{L^{k+1}\left(\left[t_{0}, t_{0}+T\right] ; L^{k+1}\left(\mathrm{~d} k_{t}\right)\right)} \\
& \quad \leq C\left(1+T^{r}\right) \epsilon\left\|u^{(m)}-u^{(m-1)}\right\|_{L^{k+1}\left(\left[t_{0}, t_{0}+T\right] ; L^{k+1}\left(\mathrm{~d} k_{t}\right)\right)}
\end{aligned}
$$

Thus, we may find $\epsilon$ small (and independent of $t_{0}$ ) so that the sequence $u^{(m)}$ converges to a solution in $L^{k+1}\left(\left[t_{0}, t_{0}+T\right] ; L^{k+1}\left(\mathrm{~d} k_{t}\right)\right)$. Uniqueness follows by standard arguments.

We now appeal to the energy bounds extend the solution to an interval of length $2 T$, as the energy at time $t_{0}+T$ is no larger than $A^{2} \epsilon$. We may thus iterate the process, finishing the proof.

\section{References}

[1] Baskin, D.: A parametrix for the fundamental solution of the Klein-Gordon equation on asymptotically de Sitter spaces. J. Funct. Anal. 259(7), 1673-1719 (2010)

[2] Baskin, D.: A Strichartz estimate for de Sitter space. In: The AMSI-ANU Workshop on Spectral Theory and Harmonic Analysis. Proc. Centre Math. Appl. Austral. Nat. Univ., vol. 44, pp. 97-104. Austral. Nat. Univ., Canberra (2010)

[3] Baskin, D.R.: Wave equations on asymptotically de Sitter spaces. PhD thesis, Stanford University (2010)

[4] Christ, M., Kiselev, A.: Maximal functions associated to filtrations. J. Funct. Anal. 179(2), 409-425 (2001)

[5] Geroch, R.: Domain of dependence. J. Math. Phys. 11, 437-449 (1970)

[6] Grigis, A., Sjöstrand, J.: Microlocal Analysis for Differential Operators. London Mathematical Society Lecture Note Series, vol. 196. Cambridge University Press, Cambridge (1994)

[7] Ginibre, J., Velo, G.: On the global Cauchy problem for some nonlinear Schrödinger equations. Ann. Inst. H. Poincaré Anal. Non Linéaire 1(4), 309-323 (1984)

[8] Hörmander, L.: Fourier integral operators. I. Acta Math. 127(1-2), 79-183 (1971)

[9] Hassell, A., Tao, T., Wunsch, J.: Sharp Strichartz estimates on nontrapping asymptotically conic manifolds. Am. J. Math. 128(4), 963-1024 (2006)

[10] Joshi, M.S., Sá Barreto, A.: Inverse scattering on asymptotically hyperbolic manifolds. Acta Math. 184(1), 41-86 (2000) 
[11] Kapitanskii, L.: Norm estimates in Besov and Lizorkin-Triebel spaces for the solutions of second-order linear hyperbolic equations. J. Math. Sci. 56(2), 23482389. doi:10.1007/BF01671936

[12] Keel, M., Tao, T.: Endpoint Strichartz estimates. Am. J. Math. 120(5), 955980 (1998)

[13] Koch, H., Tataru, D., Zworski, M.: Semiclassical $L^{p}$ estimates. Ann. Henri Poincaré 8(5), 885-916 (2007)

[14] Melrose, R.: Calculus of conormal distributions on manifolds with corners. Int. Math. Res. Notices 3, 51-61 (1992)

[15] Mazzeo, R.R., Melrose, R.: Meromorphic extension of the resolvent on complete spaces with asymptotically constant negative curvature. J. Funct. Anal. 75(2), 260-310 (1987)

[16] Marzuola, J., Metcalfe, J., Tataru, D., Tohaneanu, M.: Strichartz estimates on Schwarzschild black hole backgrounds. Commun. Math. Phys. 293(1), 3783 (2010)

[17] Mockenhaupt, G., Seeger, A., Sogge, C.D.: Local smoothing of Fourier integral operators and Carleson-Sjölin estimates. J. Am. Math. Soc. 6(1), 65-130 (1993)

[18] Pecher, H.: Nonlinear small data scattering for the wave and Klein-Gordon equation. Math. Z. 185(2), 261-270 (1984)

[19] Smith, H.F.: Spectral cluster estimates for $C^{1,1}$ metrics. Am. J. Math. 128(5), 1069-1103 (2006)

[20] Stein, E.M.: Singular integrals and differentiability properties of functions. Princeton Mathematical Series, No. 30. Princeton University Press, Princeton (1970)

[21] Strichartz, R.: Restrictions of Fourier transforms to quadratic surfaces and decay of solutions of wave equations. Duke Math. J. 44(3), 705-714 (1977)

[22] Tataru, D.: Strichartz estimates for second order hyperbolic operators with nonsmooth coefficients. II. Am. J. Math. 123(3), 385-423 (2001)

[23] Taylor, M.E.: Partial differential equations. I. Basic theory. Applied Mathematical Sciences, vol. 115. Springer, New York (1996)

[24] Tohaneanu, M.: Strichartz estimates on Kerr black hole backgrounds. Trans. Am. Math. Soc. 364, 689-702 (2012)

[25] Vasy, A.: The wave equation on asymptotically de Sitter-like spaces. Adv. Math. 223, 49-97 (2010)

[26] Yagdjian, K.: The semilinear Klein-Gordon equation in de Sitter spacetime. Discrete Contin. Dyn. Syst. Ser. S 2(3), 679-696 (2009)

[27] Yagdjian, K., Galstian, A.: Fundamental solutions for the Klein-Gordon equation in de Sitter spacetime. Commun. Math. Phys. 285(1), 293-344 (2009)

Dean Baskin

Northwestern University

Evanston, IL 60208, USA

e-mail: dbaskin@math.northwestern.edu

Communicated by Piotr T. Chrusciel.

Received: August 15, 2011.

Accepted: May 16, 2012. 